ThE Astrophysical JouRnAL, 562:279-296, 2001 November 20

(C) 2001. The American Astronomical Society. All rights reserved. Printed in U.S.A.

\title{
COMPRESSIBLE MAGNETOHYDRODYNAMIC TURBULENCE IN INTERSTELLAR PLASMAS
}

\author{
Yoram LithWick AND PeTER GoldReich \\ California Institute of Technology, MS 130-33, Pasadena, CA 91125; yoram@tapir.caltech.edu, pmg@gps.caltech.edu \\ Received 2001 June 21 ; accepted 2001 July 24
}

\begin{abstract}
Radio wave scintillation observations reveal a nearly Kolmogorov spectrum of density fluctuations in the ionized interstellar medium. Although this density spectrum is suggestive of turbulence, no theory relevant to its interpretation exists. We calculate the density spectrum in turbulent magnetized plasmas by extending the theory of incompressible magnetohydrodynamic (MHD) turbulence given by Goldreich $\&$ Sridhar to include the effects of compressibility and particle transport. Our most important results are as follows:
\end{abstract}

1. Density fluctuations are due to the slow mode and the entropy mode. Both modes are passively mixed by the cascade of shear Alfvén waves. Since the shear Alfvén waves have a Kolmogorov spectrum, so do the density fluctuations.

2. Observed density fluctuation amplitudes constrain the nature of MHD turbulence in the interstellar medium. Slow mode density fluctuations are suppressed when the magnetic pressure is less than the gas pressure. Entropy mode density fluctuations are suppressed by cooling when the cascade timescale is longer than the cooling timescale. These constraints imply either that the magnetic and gas pressures are comparable or that the outer scale of the turbulence is very small.

3. A high degree of ionization is required for the cascade to survive damping by neutrals and thereby to extend to small length scales. Regions that are insufficiently ionized produce density fluctuations only on length scales larger than the neutral damping scale. These regions may account for the excess of power that is found on large scales.

4. Provided that the thermal pressure exceeds the magnetic pressure, both the entropy mode and the slow mode are damped on length scales below that at which protons can diffuse across an eddy during the eddy's turnover time. Consequently, eddies whose extents along the magnetic field are smaller than the proton collisional mean free path do not contribute to the density spectrum. However, in MHD turbulence eddies are highly elongated along the magnetic field. From an observational perspective, the relevant length scale is that transverse to the magnetic field. Thus, the cutoff length scale for density fluctuations is significantly smaller than the proton mean free path.

5. The Alfvén mode is critically damped at the transverse length scale of the proton gyroradius and thus cascades to smaller length scales than either the slow mode or the entropy mode.

Subject headings: ISM: kinematics and dynamics - MHD - turbulence

\section{INTRODUCTION}

Diffractive scintillations of small angular diameter radio sources indicate that the interstellar electron density spectrum on length scales of $10^{8}-10^{10} \mathrm{~cm}$ is nearly Kolmogorov, i.e., rms density fluctuations across a length scale $\lambda$ are nearly proportional to $\lambda^{1 / 3}$. They also establish that there are large variations in the amplitude of the density spectrum along different lines of sight.

Rickett $(1977,1990)$ and Armstrong, Rickett, \& Spangler (1995) review the observations of diffractive scintillation and their interpretation. They also discuss refractive scintillations and dispersion measure fluctuations, which probe density fluctuations on scales larger than the diffractive scales. Nondiffractive measurements tend to indicate that the Kolmogorov spectrum extends to much larger scales. However, we focus primarily on diffractive measurements because they are much more sensitive.

Density fluctuations that obey the Kolmogorov scaling occur in homogeneous subsonic hydrodynamic turbulence. They are due to the entropy mode, a zero-frequency isobaric mode whose density fluctuations are offset by temperature fluctuations. Since subsonic turbulence is nearly incompressible, the velocity fluctuations follow Kolmogorov's scaling. To a good approximation, the entropy mode is passively mixed by the velocity field, so it also conforms to the Kolmogorov spectrum. ${ }^{1}$ Density fluctuations in Earth's atmosphere, which cause stars to twinkle, obey the Kolmogorov scaling. They arise from the passive mixing of the entropy mode.

The electron density spectrum in the interstellar medium cannot be explained by hydrodynamic turbulence. ${ }^{2}$ Because the medium is ionized, magnetic effects must be accounted for. This is evident since the length scales probed by diffractive scintillations are smaller than the collisional mean free paths of both electrons and protons. If the magnetic field

\footnotetext{
${ }^{1}$ Density fluctuations due to the Reynolds stress scale as $\lambda^{2 / 3}$. In addition, the dissipation of turbulent kinetic energy yields entropy fluctuations. The ratio of the corresponding density fluctuations to the mean density is comparable to the square of the Mach number at the length scale of interest; hence, these density fluctuations are also proportional to $\lambda^{2 / 3}$.

${ }^{2}$ Charge neutrality is maintained on diffractive scales, so electron density fluctuations include compensating fluctuations in the density of positive ions.
} 
were negligible, freely streaming plasma would wipe out density fluctuations at diffractive scales. In the presence of a magnetic field, electrons and protons are tied to field lines at the scale of their gyroradii. For typical interstellar field strengths, these gyroradii are smaller than the diffractive scales. A magnetic field thus impedes the plasma from streaming across field lines and allows the turbulent cascade and the associated density fluctuations to reach very small scales across the field lines before dissipating. Therefore, a theory for compressible turbulence in magnetized plasmas is required to explain the observed density spectra. Our objective is to develop this theory.

Until now, the only description of density fluctuations in interstellar plasmas was by Higdon (1984, 1986). These papers, while prescient, preceded a theory for incompressible MHD turbulence and therefore did not account for the full dynamics of the cascade. We compare Higdon's theory with ours in $\S 10$.

Our compressible theory extends the theory of incompressible MHD turbulence given by Goldreich \& Sridhar (1995) by including a slightly compressible slow mode and a passive entropy mode. We also consider kinetic effects: on sufficiently short length scales, the mean free paths of the particles are significant, and the equations of compressible MHD must be modified. This is especially important for damping.

In a future paper we will apply the theory developed here to estimate amplitudes for density fluctuations produced in supernova shocks, $\mathrm{H}$ II regions, stellar winds, and thermally unstable regions. These are then compared to scattering measures observed along different lines of sight.

Before considering compressible turbulence, we discuss incompressible MHD turbulence, focusing on issues that are important for the compressible case.

\section{INCOMPRESSIBLE MHD TURBULENCE}

Goldreich \& Sridhar $(1995,1997)$ propose a picture of the dynamics of incompressible strong MHD turbulence and describe the power spectra of Alfvén waves, slow waves, and passive scalars. We extend their picture to cover additional features such as the parallel cascades of both slow waves and passive scalars. Throughout this paper, "parallel" and "transverse" refer to the orientation relative to the "local mean magnetic field," which is the magnetic field averaged over the scale of interest. Our discussion of incompressible MHD turbulence, while somewhat lengthy, is important for understanding the extension to compressible turbulence that follows.

Consider a uniform unperturbed plasma with an embedded magnetic field. Turbulence is excited at the MHD outer scale, $L_{\mathrm{MHD}}$, by random and statistically isotropic forcing, with rms velocity fluctuations and rms magnetic field fluctuations (in velocity units) that are comparable to the Alfvén speed, $v_{\mathrm{A}} \cdot{ }^{3}$

As the turbulence cascades from the MHD outer scale to smaller scales, power concentrates in modes with increasingly transverse wavevectors. The inertial range velocity spectrum applies to length scales below $L_{\mathrm{MHD}}$ but above the

\footnotetext{
${ }^{3}$ The forcing fluctuations may also be less $v_{\mathrm{A}}$, in which case $L_{\mathrm{MHD}}$ would be defined as the length scale at which the fluctuations extrapolate to $v_{\mathrm{A}}$.
}

dissipation scale. It is anisotropic and is characterized by

$$
\begin{gathered}
v_{\lambda_{\perp}}=v_{\mathrm{A}}\left(\frac{\lambda_{\perp}}{L_{\mathrm{MHD}}}\right)^{1 / 3}, \\
\Lambda_{\|}=\lambda_{\perp}^{2 / 3} L_{\mathrm{MHD}}^{1 / 3} .
\end{gathered}
$$

The inertial range magnetic field spectrum is identical. Here $\lambda_{\perp}$ is the length scale transverse to the local mean magnetic field, $v_{\lambda_{\perp}}$ is the rms velocity fluctuation across $\lambda_{\perp}$, and $\Lambda_{\|}$is the length scale parallel to the local mean magnetic field across which the velocity fluctuation is $v_{\lambda_{\perp}}$. We interpret $\Lambda_{\|}$ as the elongation along the magnetic field of an "eddy" that has a size $\lambda_{\perp}$ transverse to the magnetic field; it is not an independent variable but is a function of $\lambda_{\perp}$. Deep within the inertial range, where $\lambda_{\perp} \ll L_{\mathrm{MHD}}$, eddies are highly elongated along the magnetic field: $\Lambda_{\|} \gg \lambda_{\perp}$. In the following subsections we explain the physics underlying the spectrum and consider some of the implications.

\subsection{Alfvén Wave Spectrum}

Arbitrary disturbances can be decomposed into Alfvén waves and slow waves. Appendix A summarizes the properties of these waves in the more general case of compressible MHD. In incompressible MHD, Alfvén waves and slow waves are usually referred to as shear Alfvén waves and pseudo-Alfvén waves, but the former designation is more convenient for making the connection with compressible MHD.

Our understanding of the MHD turbulence is based on two facts: (1) MHD wave packets propagate at the Alfvén speed either parallel or antiparallel to the local mean magnetic field, and (2) nonlinear interactions are restricted to collisions between oppositely directed wave packets. These facts imply that in encounters between oppositely directed wave packets, each wave packet is distorted as it follows field lines perturbed by its collision partner. A wave packet cascades when the field lines along which it is propagating have spread by a distance comparable to its transverse size.

Alfvén waves have quasi two-dimensional velocity and magnetic field fluctuations that are confined to planes perpendicular to the local mean magnetic field. As their more complete name "shear Alfvén" implies, they dominate the shear of the mapping of planes transverse to the local mean magnetic field produced by field line wander. Thus, Alfvén waves control the dynamics of MHD cascades; slow waves may be ignored when considering the dynamics of Alfvén waves.

In strong MHD turbulence the cascade time of an Alfvén wave packet is comparable to its travel time across the parallel length of a single oppositely directed Alfvén wave packet of similar size. Goldreich \& Sridhar (1995) refer to this balance of timescales as "critical balance." It relates the parallel size of a wave packet, $\Lambda_{\|}$, to its transverse size, $\lambda_{\perp}$. Wave packets of transverse size $\lambda_{\perp}$ cascade when the field lines they follow wander relative to each other by a transverse distance $\lambda_{\perp}$. Critical balance implies that this occurs over a parallel distance $\Lambda_{\|}$.

The Alfvén wave spectrum is given by equations (1) and (2), with $v_{\lambda_{\perp}}$ referring to the velocity fluctuations of the Alfvén waves. It is deduced from two scaling arguments: (1) Kolmogorov's argument that the cascade time $t_{\lambda_{\perp}} \simeq \lambda_{\perp} / v_{\lambda_{\perp}}$ leads to an energy cascade rate, $v_{\lambda_{\perp}}^{2} / t_{\lambda_{\perp}} \simeq v_{\lambda_{\perp}}^{3} / \lambda_{\perp}$, which is independent of length scale; and (2) the "critical balance" 
assertion that the linear wave period that characterizes the Alfvén waves in a wave packet is comparable to the nonlinear cascade time of that wave packet, i.e., $v_{\mathrm{A}} / \Lambda_{\|} \simeq 1 / t_{\lambda_{\perp}}$.

Before considering slow waves in MHD turbulence, we discuss two topics that are governed by the dynamics of Alfvén waves only: eddies and passive scalars.

\subsection{Eddies}

Because of their transverse polarization, Alfvén waves are responsible for the wandering of magnetic field lines. A snapshot of wandering field lines is shown in Figure 1. Each of these field lines passes through a localized region of size $\lambda_{\perp}$ in one plane transverse to the mean magnetic field. Away from this plane the bundle of field lines diverges as a result of the differential wandering of the individual lines. At a second plane, the bundle's cross-sectional area has approximately doubled. Critical balance implies that the distance to this second plane is comparable to the parallel wavelength that characterizes the bundle, $\Lambda_{\|}$. As the bundle spreads, other field lines, not depicted, enter from its sides. In general, the neighboring field lines of any individual field line within a region of transverse size $\lambda_{\perp}$ change substantially over a parallel distance of order $\Lambda_{\|}$. It is natural to think of $\Lambda_{\|}$as the parallel size of an "eddy" that has transverse size $\lambda_{\perp}$. Two eddies with the same transverse length scale that are separated by a parallel distance greater than

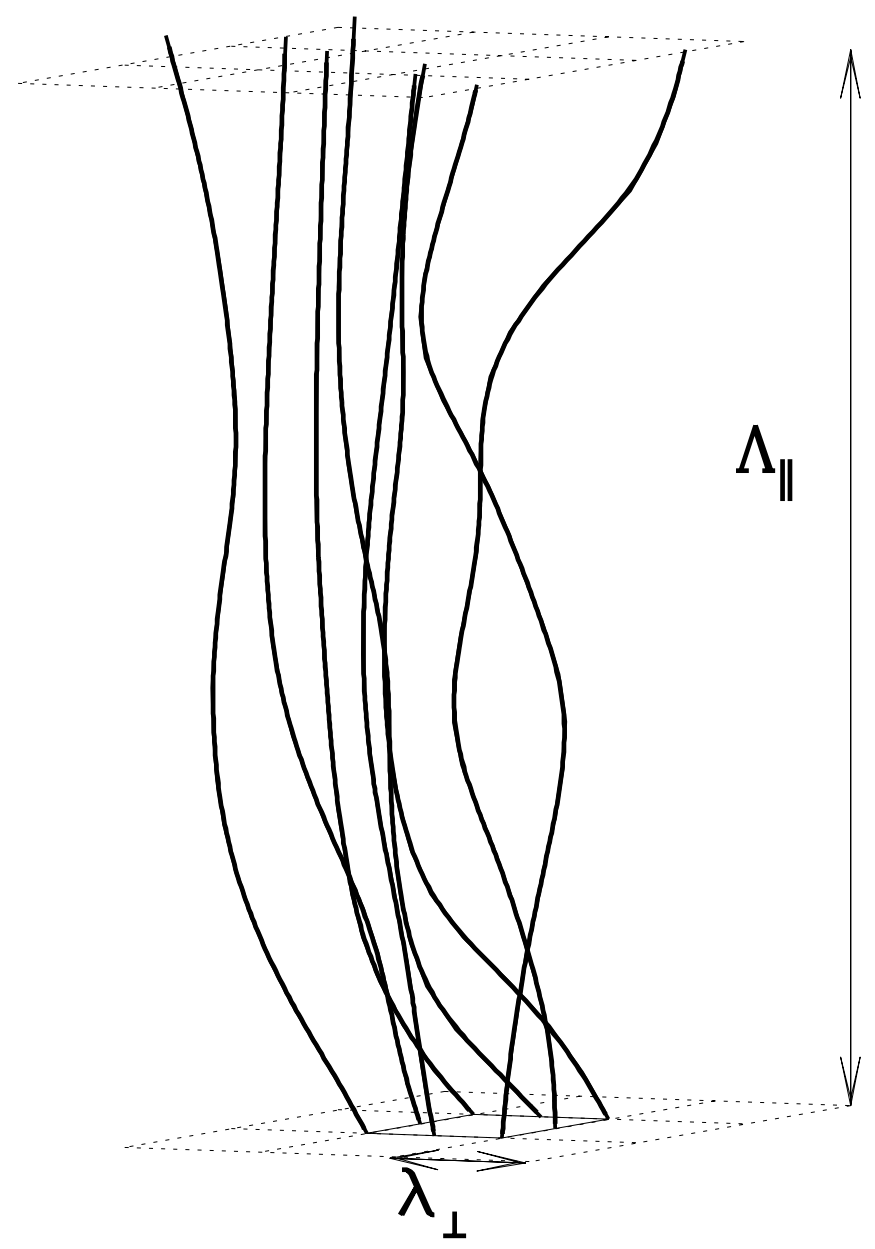

FIG. 1.-Wandering of magnetic field lines. A field line bundle of transverse size $\lambda_{\perp}$ diverges after a parallel distance $\Lambda_{\|}$, where $\Lambda_{\|}$is the parallel size of an eddy (eq. [2]) as determined by critical balance. their $\Lambda_{\|}$incorporate different field lines and hence are statistically independent. Eddies are distinct from wave packets. The former are rooted in the fluid, whereas the latter propagate up and down magnetic field lines at the Alfvén speed.

Aside from their anisotropy, eddies in MHD turbulence are similar to those in hydrodynamic turbulence. They are spatially localized structures with characteristic velocity fluctuations and lifetimes. The rms velocity difference between two points is determined by the smallest eddy that contains both. Different eddies of a given size are statistically independent. The three-dimensional spectrum for rms velocity fluctuations across transverse length scales $\lambda_{\perp}$ and parallel length scales $\lambda_{\|}$is

$$
\begin{aligned}
v_{\lambda_{\perp}, \lambda_{\|}}= & v_{\mathrm{A}} \\
& \times \begin{cases}\left(\frac{\lambda_{\perp}}{L_{\mathrm{MHD}}}\right)^{1 / 3} & \text { for } \lambda_{\|} \ll \Lambda_{\|}, \\
\left(\frac{\lambda_{\|}}{L_{\mathrm{MHD}}}\right)^{1 / 2}=\left(\frac{\lambda_{\perp}}{L_{\mathrm{MHD}}}\right)^{1 / 3}\left(\frac{\lambda_{\|}}{\Lambda_{\|}}\right)^{1 / 2} & \text { for } \lambda_{\|} \gg \Lambda_{\|} .\end{cases}
\end{aligned}
$$

There is negligible additional power within an eddy on parallel length scales smaller than $\Lambda_{\|}$, so for $\lambda_{\|} \ll \Lambda_{\|}\left(\lambda_{\perp}\right)$, $v_{\lambda_{\perp}, \lambda_{\|}}=v_{\lambda_{\perp}}$. For $\lambda_{\|} \gg \Lambda_{\|}\left(\lambda_{\perp}\right)$, the smallest eddy that contains both $\lambda_{\perp}$ and $\lambda_{\|}$has a transverse length scale $\lambda_{\perp}^{\prime}$ that satisfies $\Lambda_{\|}\left(\lambda_{\perp}^{\prime}\right)=\lambda_{\|}$. The velocity fluctuation of this eddy is obtained by solving this equation for $\lambda_{\perp}^{\prime}$ (eq. [2]) and inserting this $\lambda_{\perp}^{\prime}$ in equation (1). Contours of the threedimensional spectrum are plotted in Figure 2. Each contour represents eddies of a characteristic size.

Maron \& Goldreich (2001) give the three-dimensional spectrum in Fourier space. Since eddies that are separated by more than $\Lambda_{\|}$are statistically independent, the power spectrum at a fixed transverse wavenumber $k_{\perp}$ is indepen-

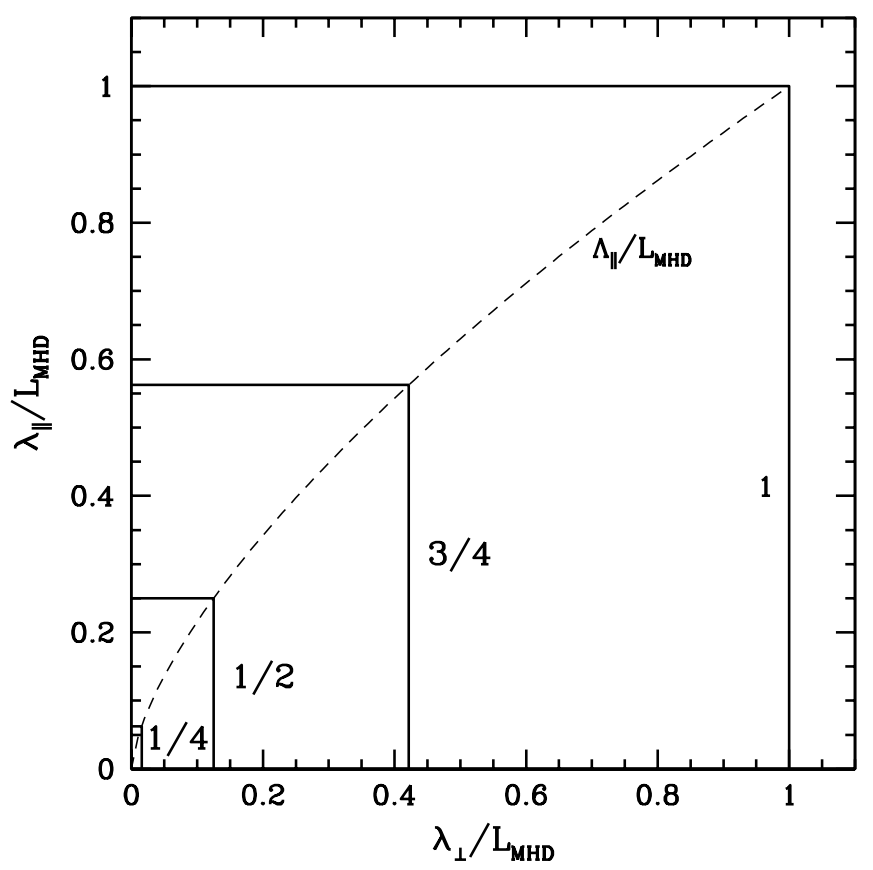

FIG. 2.-Three-dimensional spectrum. Contours of constant $v_{\lambda_{1}, \lambda_{\|}}$(eq. [3]), labeled by the value of $v_{\lambda_{1}, \lambda_{\|}} / v_{\mathrm{A}}$. Along the $\lambda_{\perp}$-axis, $v_{\lambda_{\perp}, 0} / v_{\mathrm{A}}=$ $\left(\lambda_{\perp} / L_{\mathrm{MHD}}\right)^{1 / 3}$; along the $\lambda_{\|}$-axis, $v_{0, \lambda_{\|}} / v_{\mathrm{A}}=\left(\lambda_{\|} / L_{\mathrm{MHD}}\right)^{1 / 2}$. 
dent of the parallel wavenumber $k_{\|}$in the corresponding region of Fourier space, i.e., where $k_{\|}^{-1} \gtrsim \Lambda_{\|}\left(k_{\perp}^{-1}\right)$.

The turbulent cascade is generally viewed as "proceeding" from larger eddies to smaller eddies as this is the direction of energy transfer. However, smaller eddies cascade many times in the time that a large eddy cascades. This is particularly important in turbulent mixing. Consider the evolution of two fluid elements whose initial separation is larger than the dissipation scale. On cascade timescale $t_{\lambda_{1}}$, their transverse separation will random walk a distance $\lambda_{\perp}$ as the result of the cascade of smaller eddies. Therefore, on a timescale comparable to an eddy's cascade time, the transverse locations of its component fluid elements, whose sizes may be considered to be comparable to the dissipation scale, are completely randomized. Moreover, since mixing at the dissipation scale causes neighboring fluid elements to be rapidly homogenized, transverse smoothing of the eddy occurs on the timescale that it cascades. Rapid transverse mixing in MHD turbulence is similar to the more familiar isotropic mixing in hydrodynamic turbulence.

\subsection{Passive Scalar Spectrum}

A passive scalar, $\sigma$, satisfies the continuity equation $(\partial / \partial t$ $+v \cdot \nabla) \sigma=0$ and does not affect the fluid's evolution. It could represent, for example, the concentration of a contaminant. We consider the spectrum of a passive scalar mixed by the Alfvén wave cascade. These considerations are important for our subsequent investigation of compressible turbulence. They are also helpful for understanding the slow wave spectrum. We discuss the passive scalar spectrum both in the inertial range and also below the scale at which the Alfvén wave spectrum is cut off.

\subsubsection{Passive Scalar Spectrum in the Inertial Range}

As we show in this subsection, the transverse spectrum of the passive scalar in the inertial range is

$$
\sigma_{\lambda_{\perp}} \propto \lambda_{\perp}^{1 / 3}
$$

where $\lambda_{\perp}$ is the length scale transverse to the local mean magnetic field and $\sigma_{\lambda_{\perp}}$ is the rms fluctuation in the passive scalar across $\lambda_{\perp}$. The passive scalar parallel spectrum is the same as the Alfvén wave parallel spectrum given in equation (2), where $\Lambda_{\|}$is now to be interpreted as the length scale parallel to the local mean magnetic field across which the passive scalar fluctuation is $\sigma_{\lambda_{\perp}}$.

Mixing of the passive scalar is due to Alfvén waves. Slow wave mixing is negligible. This is because transverse velocity gradients are much larger than parallel ones in MHD turbulence. Thus, Alfvén waves, whose velocity fluctuations are perpendicular to the magnetic field, are much more effective at mixing than slow waves, whose velocity fluctuations are nearly parallel to the magnetic field. The transverse cascade arises from the shuffling of field lines as Alfvén waves propagate through the fluid.

The transverse spectrum (eq. [4]) follows from the Kolmogorov-like hypothesis that the cascade rate of the "energy" in the scalar field is independent of length scale, i.e., $\sigma_{\lambda_{\perp}}^{2} / t_{\lambda_{\perp}}$ is constant, where $t_{\lambda_{\perp}}$ is the passive scalar cascade time, which is assumed to be proportional to the cascade time of Alfvén waves. Comparing this with the constancy of the kinetic energy cascade rate, $v_{\lambda_{\perp}}^{2} / t_{\lambda_{1}}$, we conclude that $\sigma_{\lambda_{\perp}} \propto v_{\lambda_{1}}$, which implies equation (4). A similar argument holds for the cascade of a passive scalar in hydrodynamic turbulence (e.g., Tennekes \& Lumley 1972).
The parallel cascade of a passive scalar is more subtle. It might appear that a passive scalar cannot cascade along field lines since, neglecting dissipation, both the scalar and the magnetic field are frozen to the fluid, and thus the scalar must be frozen to field lines. In that case there certainly could not be a parallel cascade. If the scalar were injected on large scales, then fluctuations with smaller wavelengths along the magnetic field would not be generated. However, dissipation cannot be neglected. It is an essential part of MHD turbulence, as it is of hydrodynamic turbulence. For example, the description of turbulent mixing in $\S 2.2$ depends crucially upon small-scale dissipation.

Perhaps the best way to understand the parallel cascade is to consider mixing on the transverse length scale $\lambda_{\perp}$ within two planes that are perpendicular to the local mean magnetic field and are separated by a parallel distance larger than $\Lambda_{\|}$. Velocity fluctuations within the two planes are statistically independent. This is evident because a bundle of field lines cannot be localized within a transverse distance $\lambda_{\perp}$ over a parallel separation greater than $\Lambda_{\|}$. Even a pair of fluid elements, one in each plane, that are initially on the same field line are mixed into two regions with different values of passive scalar concentration. It follows that the parallel cascade of the passive scalar also obeys equation (2).

\subsubsection{Passive Scalar Spectrum below Alfvén Wave Cutoff}

A passive scalar cascade may extend below the transverse scale at which the MHD cascade is cut off. Mixing on these scales is driven by fluid motions at $\lambda_{\text {cutoff }}$, which results in a scale-independent mixing time equal to the cascade time at $\lambda_{\text {cutoff }}$. This yields

$$
\sigma_{\lambda_{\perp}}=\text { constant }, \lambda_{\perp}<\lambda_{\text {cutoff }} .
$$

A similar argument applies in hydrodynamic turbulence. Tennekes \& Lumley (1972) call this regime in hydrodynamic turbulence the "viscous convective subrange."

\subsection{Slow Wave Spectrum}

The slow wave spectrum is the same as that of the Alfvén waves. It is given by equations (1) and (2), with $v_{\lambda_{\perp}}$ referring to the velocity fluctuations of the slow waves. This is a consequence of the similar kinematics of slow waves and shear Alfvén waves and the fact that both are cascaded by shear Alfvén waves.

Slow waves obey the same linear wave equation as Alfvén waves, and to lowest nonlinear order they travel up and down the local mean magnetic field lines at the Alfvén speed just as Alfvén waves do. However, the dynamics of the MHD cascade is controlled by the Alfvén waves (Goldreich \& Sridhar 1997; Maron \& Goldreich 2001) because their velocity and magnetic field fluctuations are perpendicular to the local mean magnetic field, whereas those of the slow waves are nearly parallel to it. Since perpendicular gradients are much larger than parallel ones in the MHD cascade, Alfvén waves are much more effective at mixing than are slow waves. Hence, Alfvén waves cascade both themselves and slow waves, whereas slow waves cascade neither. ${ }^{4}$

The transverse mixing of the slow waves by Alfvén waves is analogous to the mixing of a passive scalar. As discussed

\footnotetext{
${ }^{4}$ We are assuming that Alfvén and slow waves have comparable strengths at a given length scale.
} 
in $\S 2.3$, a passive scalar assumes the same inertial range spectrum as that of the velocity field that is responsible for its mixing. Thus, equation (1) is also applicable to the velocity fluctuations of the slow waves.

Similarly, the parallel cascade of slow waves is analogous to the parallel cascade of a passive scalar. Since Alfvén waves cascade in the time they move a distance $\Lambda_{\|}$, slow waves separated by this distance are independently mixed. Thus, equation (2) also applies to slow waves. There is, however, a conceptual difference between the parallel cascades of the passive scalar and of the slow mode. In the absence of dissipation a passive scalar is frozen to field lines, whereas slow mode wave packets travel along them at the Alfvén speed. A passive scalar has a parallel cascade because Alfvénic fluctuations are statistically independent within two transverse planes frozen in the fluid and separated by $\Lambda_{\|}$. The parallel cascade of slow waves occurs because two transverse planes separated by $\Lambda_{\|}$that are travelling at the Alfvén speed in the same direction experience uncorrelated sequences of distortions suffered as a result of interactions with oppositely directed Alfvén waves. Nevertheless, these two requirements are both satisfied in the MHD cascade when the transverse planes are separated by a distance greater than $\Lambda_{\|}$, and so the passive scalar and the slow mode have the same parallel spectrum. Whereas passive scalar mixing is due to eddies, slow wave mixing may be thought of as due to "travelling eddies," i.e., eddies that travel up and down the magnetic field at the Alfvén speed.

\subsection{Numerical Simulations}

Numerical simulations offer some support for the above description of incompressible MHD turbulence. Those by Cho \& Vishniac (2000b) support both equations (1) and (2), and those by Müller \& Biskamp (2000) support equation (1). However, although the simulations of Maron \& Goldreich (2001) support equation (2), they yield $v_{\lambda_{\perp}} \propto \lambda_{\perp}^{1 / 4}$ instead of equation (1). Because the simulations of Maron \& Goldreich (2001) are stirred highly anisotropically, whereas those of Cho \& Vishniac (2000b) and Müller \& Biskamp (2000) are stirred isotropically, it is not clear whether these disparate results conflict. Maron \& Goldreich (2001) speculate that the discrepancy between their spectrum and the scaling prediction of Goldreich \& Sridhar (1995) results from intermittency. In any case, we expect that the physical picture of a critically balanced cascade, which underlies Goldreich \& Sridhar's (1995) description of MHD turbulence, remains valid. Even if the spectrum is proportional to $\lambda_{\perp}^{1 / 4}$, we expect that the results of this investigation, which assumes a spectrum proportional to $\lambda_{\perp}^{1 / 3}$, would not be significantly altered.

The simulations of Maron \& Goldreich (2001) confirm that a passive scalar has the same transverse spectrum as that of Alfvén waves, although both are proportional to $\lambda_{\perp}^{1 / 4}$. They also indicate that the parallel cascade of a passive scalar conforms to equation (2).

Maron \& Goldreich (2001) present results from simulations of the interaction between oppositely directed slow and Alfvén waves; the slow waves cascade, whereas the Alfvén waves do not. They also compute the spectrum of slow waves in a simulation of MHD turbulence and find that its transverse and longitudinal behavior matches that of the Alfvén wave spectrum.

\subsubsection{Kolmogorov Constants}

Scaling arguments do not yield values for the "Kolmogorov constants," the order-unity multiplicative constants of the spectrum. However, they can be obtained from simulations. We define them such that equation (1) remains valid, i.e., we take $L_{\text {MHD }}$ to be the separation at which the rms velocity difference is equal to, or extrapolates to, $v_{\mathrm{A}}$. Two Kolmogorov constants, $M_{\|}$and $M_{t}$, are needed in this paper:

$$
\begin{gathered}
M_{\|} \equiv \frac{v_{\mathrm{A}}}{v_{\lambda_{\perp}}} \frac{\lambda_{\perp}}{\Lambda_{\|}} \Rightarrow \Lambda_{\|}=M_{\|}^{-1} \lambda_{\perp}^{2 / 3} L_{\mathrm{MHD}}^{1 / 3}, \\
M_{t} \equiv \frac{v_{\mathrm{A}} t_{\lambda_{\perp}}}{\Lambda_{\|}} .
\end{gathered}
$$

In these definitions, $\lambda_{\perp}$ and $\Lambda_{\|}$are the inverses of the wavenumbers transverse and parallel to the local mean magnetic field, and $t_{\lambda_{\perp}}$ is the cascade time of waves with transverse wavenumber $\lambda_{\perp}^{-1}$. From the numerical simulations of Maron \& Goldreich (2001),

$$
M_{\|} \simeq 3.4, \quad M_{t} \simeq 1.4 \text {. }
$$

Because these simulations yield a transverse spectrum that is proportional to $\lambda_{\perp}^{1 / 4}$ instead of $\lambda_{\perp}^{1 / 3}$, the resulting "Kolmogorov constants" are not truly constant.

\section{COMPRESSIBLE TURBULENCE: OVERVIEW}

Our primary concern is interstellar scintillation, which is affected by electron density fluctuations on very small scales, typically $10^{8}-10^{10} \mathrm{~cm}$ for diffractive scintillation. In the remainder of this paper we calculate the spectrum of density fluctuations that results from compressible turbulence in magnetized plasmas with parameters appropriate to the interstellar medium. Throughout, we consider plasmas that have more ions than neutrals and that have $1 \lesssim \beta<\infty$, where $\beta$ is the ratio of the thermal pressure to the mean magnetic pressure: $\beta \equiv 8 \pi p / B^{2}=2 c_{T}^{2} / v_{\mathrm{A}}^{2}$. Here $c_{T} \equiv(p / \rho)^{1 / 2}$ is the isothermal sound speed, $v_{\mathrm{A}}$ is the Alfvén speed, $p$ is the thermal pressure, $B$ is the magnetic field strength, and $\rho$ is the mass density. ${ }^{5}$ The incompressible limit corresponds to $\beta=\infty$.

On the length scales that we consider, compressible MHD is a good approximation for the dynamics of the ionized interstellar medium. Kinetic effects, where important, may be accounted for by simple modifications to the MHD equations. Therefore, we turn our attention to turbulence in compressible MHD.

The turbulent velocity spectrum in compressible MHD is approximately the same as the turbulent velocity spectrum in incompressible MHD because the Alfén mode remains incompressible in a compressible medium and the slow mode is only slightly compressible. Thus, the velocity spectrum for both of these modes is given by equations (1) and (2). Appendix A summarizes the properties of the relevant modes in compressible MHD.

There are two additional modes in compressible MHD that are not present in incompressible MHD. One of these is the fast mode. However, as long as $\beta \gtrsim$ few, the fast mode is essentially a sound wave. Its phase speed is larger than the phase speed of either the Alfvén mode or the slow mode,

\footnotetext{
${ }^{5}$ We briefly discuss plasmas with $\beta<1$ in $\S 11$.
} 
and so the fast mode does not couple to them. Thus, we ignore the fast mode. An analogous approximation is often made in subsonic hydrodynamic turbulence, where sound waves have little influence on the turbulent cascade. Some support for our neglect of the fast mode is provided by numerical simulations. Balsara \& Pouquet (1999) show that in simulations of turbulence with Mach number of order unity, the compressible component of the velocity is significantly smaller than the solenoidal component; they argue that this is because shocks dissipate much of the irrotational component of the velocity.

The second mode that is present only in compressible MHD is the entropy mode. When turbulent motions are adiabatic, the entropy mode is a zero-frequency mode with unperturbed pressure and with density perturbation offset by temperature perturbation (see Appendix A). In hydrodynamics, simple scaling arguments show that the entropy mode does not affect the dynamics of the fluid (i.e., the fluid obeys the incompressible equations of motion) provided that fluid motions are subsonic and that density fluctuations are smaller than the mean density; these arguments carry over directly to MHD (e.g., Higdon 1986 and references therein). Furthermore, since the entropy of any fluid element is conserved in the inertial range of adiabatic turbulence, the mixing of the entropy mode is identical to the mixing of a passive scalar (see eq. [4]), yielding the transverse spectrum

$$
s_{\lambda_{\perp}} \propto \lambda_{\perp}^{1 / 3},
$$

where $s_{\lambda_{\perp}}$ is the rms entropy fluctuation across $\lambda_{\perp}$. The parallel spectrum is given by equation (2). Analogously, in hydrodynamic turbulence the effect of the entropy mode on turbulent motions can often be neglected, and the entropy mode is mixed as a passive scalar (Monin \& Yaglom 1971).

Based on the above discussion, there are two sources of density fluctuations on small scales: the slow mode and the entropy mode. Since the slow mode density perturbation is proportional to its velocity perturbation, and since the entropy mode density perturbation is proportional to its entropy perturbation, both yield a Kolmogorov spectrum of density perturbations:

$$
n_{\lambda_{\perp}} \propto \lambda_{\perp}^{1 / 3} .
$$

In the remainder of this paper, we investigate the density spectrum in more detail. We calculate the density spectrum on diffractive length scales for given values of the number density, $n$, and outer scale, $L_{\text {out }}$. Typically, in regions of the interstellar medium that are relevant for scintillation, $1<n<100 \mathrm{~cm}^{-3}$. Values of $L_{\text {out }}$ are more uncertain, although it is likely that $L_{\text {out }}$ is typically within a few orders of magnitude of $1 \mathrm{pc}$. It is also plausible that the value of $L_{\text {out }}$ is related to that of $n$. In a future paper we will discuss in much more detail the values of these parameters in turbulent regions of the ionized interstellar medium, e.g., in supernova shocks, $\mathrm{H}$ iI regions, and stellar winds.

A naive guess for the resulting density fluctuation at the length scale $\lambda_{\perp}$ is $n_{\lambda_{\perp}}=n\left(\lambda_{\perp} / L_{\text {out }}\right)^{1 / 3}$, which we refer to as the fiducial spectrum. However, there are a number of physical effects that suppress the small-scale density spectrum in the interstellar medium relative to the fiducial spectrum.

When considering scintillation observations, it is the transverse (not parallel) length scale that is relevant. Each line of sight crosses regions with different orientations of the local mean magnetic field. The observational effects of the parallel length scale are washed out if the orientation varies by an angle greater than a tiny ratio: $\lambda_{\perp} / \Lambda_{\|}$at the diffractive scale. Since the variation in angle due to eddies on scales larger than the diffractive scale is $\lambda_{\perp} / \Lambda_{\|} \propto \lambda_{\perp}^{1 / 3}$, it increases with scale, so these eddies render the parallel length scale unobservable. As a result, we frequently refer to the transverse length scale as, simply, the length scale.

Because of the multitude of special length scales involved, we organize the discussion by decreasing length scale. We begin at the outer scale and proceed down to the smallest scales relevant to interstellar scintillation, while considering the effects of the Alfven mode, the slow mode, and the entropy mode simultaneously. Since most of the relevant length scales in the interstellar medium have a similar dependence on the background density (they decrease with increasing density), the ordering of length scales is fairly universal. The length scales that we consider are the outer scale, the MHD scale, the cooling scale, the collisionless scale of the neutrals, the collisionless scale of the ions, and the proton gyroscale. We conclude with a summary of the most important effects. Table 1 lists the length scales that are used in this paper.

TABLE 1

\begin{tabular}{|c|c|}
\hline Symbol & Description \\
\hline$\lambda_{\perp} \ldots \ldots \ldots$ & Length scale transverse to the mean magnetic field; it is comparable to the "observed" length scale \\
\hline$\lambda_{\|} \ldots \ldots \ldots$ & Length scale parallel to the mean magnetic field \\
\hline$\Lambda_{\|} \ldots \ldots \ldots$ & Parallel size of an eddy; it is a function of $\lambda_{\perp}$ (eq. [2]) \\
\hline$L_{\text {out }}^{\prime \prime} \ldots \ldots$ & $\lambda_{\perp}=L_{\text {out }}$ at the outer scale \\
\hline$L_{\mathrm{MHD}} \cdots \cdots$ & $\lambda_{\perp}=L_{\mathrm{MHD}}$ at the MHD scale \\
\hline$L_{\text {cool }} \ldots \ldots .$. & $\lambda_{\perp}=L_{\text {cool }}$ at the cooling scale \\
\hline$L_{\mathrm{H}} \ldots \ldots \ldots$ & $\lambda_{\perp}=L_{\mathrm{H}}$ at the collisionless scale of hydrogen atoms \\
\hline$L_{\mathrm{He}} \ldots \ldots \ldots$ & $\lambda_{\perp}=L_{\mathrm{He}}$ at the collisionless scale of helium atoms \\
\hline$L_{\mathrm{mfp}} \ldots \ldots .$. & Mean free path of protons and of electrons \\
\hline$L_{\text {ed }} \cdots \ldots \ldots$ & $\Lambda_{\|}=L_{\mathrm{ed}}$ at the electron diffusion scale \\
\hline$L_{\mathrm{eq}} \ldots \ldots \ldots$ & $\Lambda_{\|}^{\|}=L_{\text {eq }}$ at the electron-proton equilibration scale \\
\hline$L_{\mathrm{pd}}^{\mathrm{c}} \ldots \ldots \ldots$ & $\Lambda_{\|}^{\|}=L_{\mathrm{pd}}$ at the proton diffusion scale \\
\hline$L_{\mathrm{pd}}^{(\perp)} \ldots \ldots$ & $\lambda_{\perp}=L_{\mathrm{pd}}^{(\mathrm{pd})}$ at the proton diffusion scale \\
\hline$L_{p, \mathrm{gyr}} \cdots \cdots$ & $\lambda_{\perp}=L_{p, \mathrm{gyr}}$ at the proton gyroscale \\
\hline
\end{tabular}

Summary of Length Scales 


\section{THE OUTER SCALE AND THE MHD SCALE}

As a model for the excitation of the turbulence, we consider plasma that is stirred on an outer scale $L_{\text {out }}$ with velocity fluctuations that are of order the sound speed: $v_{L_{\text {out }}} \sim c_{s}$. The generalization from this case of Mach 1 turbulence to subsonic turbulence with arbitrary Mach number $\mathscr{M}<1$ is trivial: $L_{\text {out }}$ would be interpreted as an effective outer scale at which velocity fluctuations extrapolate to $c_{s}$. However, we focus on the Mach 1 case because it is probably the most relevant for interstellar scintillation.

If, initially, the strength of the magnetic field is negligible, then random field line stretching amplifies the mean field (Cho \& Vishniac 2000a). It is uncertain both how quickly the magnetic field is amplified and whether its energy density is amplified until it approaches equipartition with the turbulent kinetic energy density. If it does reach equipartition within a few outer scale crossing times, then $\beta \sim 1$ would be appropriate for Mach 1 turbulence. Recall that $\beta$ is the ratio of thermal pressure to magnetic pressure, or equivalently, $\beta=2 c_{T}^{2} / v_{\mathrm{A}}^{2}$. We generally assume that the mean magnetic field can approach equipartition with the gas pressure, so we think of $\beta$ as close to but a little larger than unity, although we leave its exact value unspecified.

Provided that $\beta$ is larger than unity and $\mathscr{M} \sim 1$ on scale $L_{\text {out }}$, the turbulent kinetic energy dominates the mean magnetic energy on scales just below $L_{\text {out }}$. Thus, the cascade is hydrodynamic on these length scales, and the velocity fluctuations are given by Kolmogorov's isotropic scaling: $v_{\lambda_{\perp}} \sim c_{s}\left(\lambda_{\perp} / L_{\text {out }}\right)^{1 / 3}$.

The kinetic energy density decreases toward smaller scales. At a critical scale, which we denote $L_{\mathrm{MHD}}$, the kinetic energy density is sufficiently small that it is comparable to the mean magnetic energy density: $v_{L_{\mathrm{MHD}}^{2}}^{2} \sim v_{\mathrm{A}}^{2}$, which implies that

$$
L_{\mathrm{MHD}} \sim L_{\mathrm{out}} \beta^{-3 / 2} .
$$

Below this scale, the turbulent kinetic energy density is smaller than the magnetic energy density, and the theory of compressible MHD turbulence is applicable. Note that the effects of large-scale velocity fields can be neglected when considering fluctuations on smaller scales because a largescale velocity field can be eliminated by a Galilean transformation. However, since large-scale magnetic fields cannot be transformed away, they affect the dynamics of small-scale eddies.

Hydrodynamic turbulent motions on scales slightly larger than $L_{\mathrm{MHD}}$ have speeds $\sim v_{\mathrm{A}}$. Thus, they couple to, and efficiently excite, Alfvén waves and slow waves whose phase velocities $\omega / k \approx v_{\mathrm{A}}\left(k_{z} / k\right)$ are of comparable magnitude. Provided that $\beta>1$ and $\mathscr{M} \leq 1$, fast waves, which have $\omega / k \sim c_{s}>v_{\mathrm{A}}$, are weakly excited at $L_{\mathrm{MHD}}$. In what follows, we neglect fast waves.

Alfvén waves excited at $L_{\mathrm{MHD}}$ cascade to smaller scales, and these cascading Alfvén waves in turn cascade slow waves. The spectra of both of these cascades are given by equations (1) and (2), so that

$$
v_{\lambda_{\perp}} \sim c_{s}\left(\frac{\lambda_{\perp}}{L_{\mathrm{out}}}\right)^{1 / 3}
$$

for both modes, even on scales smaller than $L_{\mathrm{MHD}}$.
We have not yet discussed density fluctuations on the scales that have been considered in this section. It is more convenient to do so in the following section.

\section{THE COOLING SCALE}

On the length scales in the interstellar medium that we have considered thus far, the radiative cooling time is shorter than the eddy turnover time. ${ }^{6}$ Consequently, in photoionized regions, turbulence on these length scales is expected to be isothermal (Sridhar 1998; Goldreich 1998; Higdon \& Conley 1998). This has only a marginal effect on the turbulent dynamics described in the previous section because isothermal Alfvén waves are identical to adiabatic Alfvén waves and isothermal slow waves are only slightly different from adiabatic slow waves. However, the entropy mode is rapidly damped in isothermal turbulence. As a consequence, small-scale density fluctuations may be significantly suppressed. There are two possible solutions to this "cooling catastrophe:" either (1) the outer scale is extremely small, small enough that the turbulence at the outer scale is nearly adiabatic; or (2) there are significant density fluctuations associated with the slow mode. However, in the latter case, the mean magnetic field must be amplified almost to equipartition with the gas pressure, so that $\beta \sim 1$. Either of these two solutions would place stringent constraints on the nature of the turbulence that is responsible for observed density fluctuations. In the following, we consider the cooling scale in more detail.

There is a critical scale for the turbulence, which we call the "cooling scale," $L_{\text {cool }}$. Above this scale the turbulence is isothermal, and below it the turbulence is adiabatic. We assume that $L_{\mathrm{cool}}<L_{\mathrm{MHD}}$ throughout this paper, except in $\S 5.2$ where we consider the case in which this inequality is reversed. The cooling scale is where the eddy turnover time, $\lambda_{\perp} / v_{\lambda_{\perp}}$, is equal to the cooling time, $t_{\text {cool }}$, i.e., with $v_{\lambda_{\perp}} \sim c_{s}\left(\lambda_{\perp} / L_{\text {out }}\right)^{1 / 3}$,

$$
\frac{L_{\mathrm{cool}}}{L_{\mathrm{out}}} \sim\left(\frac{c_{s} t_{\mathrm{cool}}}{L_{\mathrm{out}}}\right)^{3 / 2} .
$$

As we will discuss in detail in a follow-up paper, most plausible astrophysical sources of interstellar scintillation have $c_{s} t_{\text {cool }} \lesssim L_{\text {out }}$, and thus $L_{\text {cool }} \lesssim L_{\text {out }}$. In general, the kinetic power per unit mass that is dissipated in Mach 1 turbulence is $\sim c_{s}^{2} / t_{L_{\text {out }}} \sim c_{s}^{3} / L_{\text {out }}$, where $t_{L_{\text {out }}}$ is the eddy turnover time at the outer scale. The thermal power per unit mass that is required to keep the gas hot is $\sim c_{s}^{2} / t_{\text {cool }}$. Hence, only if the energy that goes into turbulent motions is as large as the total energy that goes into heating can the outer scale be as small as the cooling scale. Nonetheless, in plasmas that are thermally unstable and in isothermal shocks (assuming that the outer scale is comparable to the scale across which the density doubles), the two powers are comparable, and the cooling scale is comparable to the outer scale.

As an example, we consider an $\mathrm{H}$ in region. Photoionized plasma is thermally stable. Heating is primarily due to photoionizing photons, and cooling is primarily due to electron impact excitation of metal line transitions (e.g., Spitzer

\footnotetext{
${ }^{6}$ For definiteness, we consider plasma that is thermally stable; deviations of the temperature from its equilibrium value decay in a cooling time, $t_{\text {cool }}$.
} 
1978). ${ }^{7}$ A characteristic temperature for plasma photoionized by hot stars is $T \sim 8000 \mathrm{~K}$, which implies that the speed of sound is $c_{s} \sim 10 \mathrm{~km} \mathrm{~s}^{-1}$. The cooling time is $t_{\text {cool }} \sim 20,000\left(\mathrm{~cm}^{-3} / n\right) \mathrm{yr}$, where $n$ is the number density of electrons. Therefore,

$$
c_{s} t_{\mathrm{cool}} \sim 0.2\left(\frac{\mathrm{cm}^{-3}}{n}\right) \mathrm{pc} .
$$

A plausible value for $L_{\text {out }}$ might be the radius of an $\mathrm{H}$ II region (the "Strömgren radius"), which is $\sim 70\left(\mathrm{~cm}^{-3} / n\right)^{2 / 3}$ pc (Spitzer 1978). Therefore, with typical values of $n$ in H II regions $\left(1<n<100 \mathrm{~cm}^{-3}\right), L_{\text {out }}$ is significantly larger than $c_{s} t_{\text {cool }}$, and so the cooling scale is significantly smaller than the outer scale.

The fact that the turbulence is isothermal on large scales has important implications. Had cooling been ignored, i.e., had it been implicitly assumed that the cooling time is infinite, then one would have calculated the density spectrum as follows: there should be density fluctuations of order unity on the outer scale, implying excitation of entropy modes on the outer scale that are passively mixed by the Alfvénic turbulence to small scales. This would yield the fiducial density spectrum, $n_{\lambda_{1}} \simeq n\left(\lambda_{\perp} / L_{\text {out }}\right)^{1 / 3}$.

However, since the turbulence is isothermal on large scales, the above calculation is incorrect. The entropy mode is not passively mixed on these scales, since it is damped by cooling before it can be mixed. In the following, we consider separately two cases: first the low- $\beta$ case $\left(\beta<L_{\text {out }} / c_{s} t_{\text {cool }}\right)$, and then the high- $\beta$ case $\left(\beta>L_{\text {out }} / c_{s} t_{\text {cool }}\right)$. In each case, we show that the small-scale density fluctuations are substantially smaller than those predicted by the fiducial spectrum unless a relatively stringent condition holds: either $\beta \sim 1$ or $L_{\text {out }} \sim c_{s} t_{\text {cool }}$.

\subsection{Density Fluctuations below the Cooling Scale:

$$
1<\beta<L_{\text {out }} / c_{s} t_{\text {cool }}
$$

With regards to interstellar scintillation, the crucial difference between high- $\beta$ and low- $\beta$ turbulence lies in the compressibility of the slow mode. Since the sum of thermal pressure and magnetic pressure vanishes for the slow mode, the mode's density perturbation satisfies $n_{\lambda_{\perp}} / n \sim p_{\lambda_{\perp}} / p \sim$ $\beta^{-1} B_{\lambda_{\perp}} / B \sim \beta^{-1} v_{\lambda_{\perp}} / v_{\mathrm{A}} \sim \beta^{-1 / 2} v_{\lambda_{\perp}} / c_{s}$ (see Appendix A). Thus, assuming that the energy in slow waves is comparable to that in Alfvén waves,

$$
\frac{n_{\lambda_{\perp}}}{n} \sim \frac{1}{\sqrt{\beta}}\left(\frac{\lambda_{\perp}}{L_{\mathrm{out}}}\right)^{1 / 3}, \quad \lambda_{\perp}<L_{\mathrm{MHD}},
$$

from the slow mode, both above and below the cooling scale. Thus, slow waves can produce density fluctuations

\footnotetext{
${ }^{7}$ In this case, there is another length scale, the "photoionization scale," which we do not consider because its effects are unimportant for scintillations. This is the scale at which the eddy turnover time is comparable to the recombination time. It is slightly larger than the cooling scale because the recombination time is approximately 5 times larger than the cooling time (Spitzer 1978). On scales larger than the photoionization scale, the turbulence is in photoionization equilibrium, whereas on scales smaller than this, the ionization fraction of a fluid element is conserved. Nonetheless, the temperature of the gas is only weakly dependent on ionization fraction: metal line cooling by free electrons is exponentially dependent on temperature and thus acts as a thermostat that is only slightly affected by the ion or neutral density. Thus, the photoionization length scale does not play an important role in the density spectrum.
}

that are not much smaller than the fiducial spectrum if $\beta$ is not much larger than unity. Comparing with equation (21) below, the contribution of the slow mode to the density fluctuations at $L_{\text {cool }}$ exceeds the contribution from Reynolds stresses provided that $\beta<L_{\text {out }} / c_{s} t_{\text {cool }}$.

The compressibility of the slow mode also yields density fluctuations associated with the entropy mode. The reason is as follows. Entropy fluctuations are associated with isothermal compressible waves. Those due to isothermal slow modes are passively mixed by Alfvén waves. Mixing due to Alfvén waves with wavelengths smaller than the cooling scale takes place in less than the cooling time. It produces a spectrum of entropy modes for $\lambda_{\perp}<L_{\text {cool }}$ that gives rise to a density spectrum similar to the one given in equation (15). Nonetheless, there is some damping of entropy fluctuations when they are mixed from scales larger than the cooling scale to scales smaller than the cooling scale. We expect that the damping is of order unity. Since, as we show in the following section, the damping of the slow mode is not very large, we expect that the amplitudes of the slow and entropy modes below the cooling scale are roughly comparable. In this paper, however, we do not quantify the amount of damping of the entropy mode more precisely because it is a difficult calculation. We have attempted to quantify this damping by performing numerical simulations. However, the limited dynamical range made the interpretation of the results difficult. Nevertheless, the relative amplitude of slow and entropy waves on small scales does not appear to be observationally important; as we show in $\S 7.3$, both slow and entropy waves damp at the same scale.

\subsubsection{Slow Mode Damping at the Cooling Scale}

Although there is negligible damping of the slow mode on length scales that are either much larger or much smaller than the cooling scale, there is some damping on length scales that are comparable to the cooling scale. As we show in this section, the damping of the slow wave cascade is small because the slow mode is not very compressible. In the limit that $\beta \rightarrow \infty$, the slow mode is incompressible, and the damping disappears. In the following, we calculate the damping to first order in $1 / \beta$.

First, we calculate the damping rate of a slow wave of a fixed wavenumber. The damping rate is obtained by substituting $\tilde{c}$ (given in Appendix A, eq. [A7]) into the slow mode dispersion relation (eq. [A11]); noting that $c_{T}^{2} \equiv p / \rho=$ $\beta v_{\mathrm{A}}^{2} / 2$, this gives, to lowest order in $1 / \beta$,

$$
\frac{\omega_{i}}{v_{\mathrm{A}} k_{\|}}=\frac{-v_{\mathrm{A}} k_{\|} t_{\mathrm{cool}}}{\beta\left[1+\left(5 v_{\mathrm{A}} k_{\|} t_{\mathrm{cool}} / 2\right)^{2}\right]},
$$

where $\omega_{i}$ is the imaginary part of $\omega$, and $k_{z}$ from Appendix $\mathrm{A}$ is here relabeled $k_{\|}$. Only on length scales where $v_{\mathrm{A}} k_{\|} t_{\mathrm{cool}} \sim 1$ is this ratio nonnegligible.

To obtain the total decrement in the amplitude of the slow mode through the cooling scale, we solve a kinetic equation obtained by balancing the slow mode $k$-space energy flux with the loss rate of slow mode $k$-space energy density due to damping, i.e.,

$$
\frac{d}{d k} \frac{v_{\lambda_{\perp}}^{2}}{t_{\lambda_{\perp}}}=2 \omega_{i} \frac{v_{\lambda_{\perp}}^{2}}{k}
$$

where $\lambda_{\perp}^{-1} \equiv k_{\perp}$ is the transverse wavenumber, $t_{\lambda_{\perp}}$ is the cascade time of a slow wave with this wavenumber, and $v_{\lambda_{\perp}}$ is the velocity perturbation of the slow mode; since $k_{\perp} \simeq k$, 
we drop the $\perp$ subscript on $k$. We rewrite the kinetic equation as follows:

$$
\frac{d}{d \ln k} \ln \frac{v_{\lambda_{\perp}}^{2}}{t_{\lambda_{\perp}}}=2 \omega_{i} t_{\lambda_{\perp}}=2 M_{t} \frac{\omega_{i}}{v_{\mathrm{A}} k_{\|}}
$$

where $M_{t} \equiv v_{\mathrm{A}} k_{\|} t_{\lambda_{\perp}} \simeq 1.4$ is a Kolmogorov constant (see $\S 2.5 .1$ ). Substituting equation (16) into equation (18) and integrating, using $k_{\|} \propto k^{2 / 3}$ (eq. [2]) and $t_{\lambda_{\perp}} \propto k^{-2 / 3}$, yields the net damping through the cooling scale:

$$
\begin{aligned}
\frac{\left.\left(v_{\lambda_{\perp}} / \lambda_{\perp}^{1 / 3}\right)\right|_{\lambda_{\perp} \ll L_{\text {cool }}}}{\left.\left(v_{\lambda_{\perp}} / \lambda_{\perp}^{1 / 3}\right)\right|_{\lambda_{\perp} \gg L_{\text {cool }}}} & =\exp \left[-\frac{M_{t}}{\beta} \int_{0}^{\infty} \frac{v_{\mathrm{A}} k_{\|} t_{\mathrm{cool}}}{1+\left(5 v_{\mathrm{A}} k_{\|} t_{\mathrm{cool}} / 2\right)^{2}} \frac{d k}{k}\right] \\
& =\exp \left(-\frac{3 \pi M_{t}}{10 \beta}\right) \\
& \simeq 1-\frac{1.3}{\beta}
\end{aligned}
$$

to lowest order in $1 / \beta$. Thus, for $\beta$ slightly larger than unity, slow mode damping can be ignored.

\subsection{Density Fluctuations below the Cooling Scale:$$
\beta>L_{\text {out }} / c_{s} t_{\text {cool }}>1
$$

In this subsection only, we assume that $\beta>L_{\text {out }} / c_{s} t_{\text {cool }}$, which implies $L_{\mathrm{MHD}}<L_{\mathrm{cool}}$. In this case, the isothermal hydrodynamic turbulent cascade extends from the outer scale down to the cooling scale. Because the entropy mode is rapidly damped under isothermal conditions and hydrodynamic turbulence is incompressible to order $v_{\lambda_{\perp}} / c_{s}$, to this order there are no density fluctuations on length scales larger than $L_{\text {cool }}$. However, pressure fluctuations due to Reynolds stresses, $p_{\lambda_{\perp}} \sim \rho v_{\lambda_{\perp}}^{2}$, create second-order density and entropy fluctuations,

$$
\frac{n_{\lambda_{\perp}}}{n}=\frac{p_{\lambda_{\perp}}}{p}=s_{\lambda_{\perp}} \sim\left(\frac{v_{\lambda_{\perp}}}{c_{s}}\right)^{2}, \quad L_{\mathrm{cool}}<\lambda_{\perp}<L_{\mathrm{out}} .
$$

These entropy fluctuations couple to the entropy mode at the cooling scale. On scales below the cooling scale, the entropy mode is mixed as a passive scalar yielding

$$
\begin{aligned}
\frac{n_{\lambda_{\perp}}}{n} & \sim \frac{v_{L_{\text {cool }}}^{2}}{c_{s}^{2}}\left(\frac{\lambda_{\perp}}{L_{\mathrm{cool}}}\right)^{1 / 3} \sim\left(\frac{L_{\mathrm{cool}}}{L_{\text {out }}}\right)^{2 / 3}\left(\frac{\lambda_{\perp}}{L_{\mathrm{cool}}}\right)^{1 / 3} \\
& \sim\left(\frac{c_{s} t_{\mathrm{cool}}}{L_{\mathrm{out}}}\right)^{1 / 2}\left(\frac{\lambda_{\perp}}{L_{\mathrm{out}}}\right)^{1 / 3}, \quad \lambda_{\perp}<L_{\mathrm{cool}} .
\end{aligned}
$$

Hydrodynamic turbulence mixes the entropy mode down to $L_{\mathrm{MHD}}$. At this scale, the hydrodynamic motions turn into Alfvén and slow waves, so the entropy mode continues to be mixed below $L_{\mathrm{MHD}}$, and equation (21) remains valid. Comparing with equation (15), the density fluctuations associated with the entropy mode exceed those associated with the slow mode when $\beta>L_{\text {out }} / c_{s} t_{\text {cool }}$.

The entropy mode's density spectrum (eq. [21]) is smaller than the fiducial spectrum by the small factor $\left(c_{s} t_{\text {cool }} / L_{\text {out }}\right)^{1 / 2}$. As will be seen in the follow-up paper, it yields density fluctuations that are too small to explain much of the observed interstellar scintillation for most plausible values of $L_{\text {out }}$, such as those given by Strömgren radii of $\mathrm{H}$ II regions. There are two alternatives: either $L_{\text {out }}$ is not much larger than $c_{s} t_{\mathrm{cool}}$, or $\beta \sim 1$.

\section{THE COLLISIONLESS SCALE OF THE NEUTRALS}

In the remainder of this paper, we consider length scales that are comparable to, or smaller than, the mean free paths of the various particles; thus, kinetic effects must be considered.

In this section we calculate the damping of the turbulence by neutrals. We assume that the neutral density is smaller than the electron density, i.e., $n_{N} \lesssim n$, and that most of the ions are protons. The neutral particles that we consider are hydrogen atoms and helium atoms. The relevant length scales are the hydrogen and helium mean free paths for collisions with protons: $L_{\mathrm{H}}=5 \times 10^{13}\left(\mathrm{~cm}^{-3} / n\right) \mathrm{cm}$ and $L_{\mathrm{He}}=1.5 \times 10^{15}\left(\mathrm{~cm}^{-3} / \mathrm{n}\right) \mathrm{cm}$ (Banks 1966). The hydrogen mean free path is significantly smaller than the helium mean free path because its collisions with protons are due to resonant charge exchange.

There are two main results for this section:

1. Only if the neutral fraction is sufficiently small (eq. [49]) can the cascade survive on length scales smaller than $L_{\mathrm{H}}$. If the neutral fraction does not satisfy this condition, then all three modes (Alfvén, slow, and entropy) are damped at the same length scale.

2. Regions where the cascade does not survive contribute to an excess of density fluctuations on large scales. This might explain observations that detect an excess of power in large-scale density fluctuations.

The organization of the calculation is as follows. First, the change in wave frequency due to neutrals is calculated. Second, we consider the effect of the frequency change on the cascade. Third, we consider the cases in which the neutrals are hydrogen atoms and in which they are helium atoms. Although helium atoms have a larger mean free path, we consider them after neutral hydrogen atoms because they are of lesser importance. Finally, we consider regions where neutral damping terminates the cascade.

A similar, though less detailed, calculation is performed by Goldreich \& Sridhar (1995) for the case in which the neutrals are hydrogen atoms.

\subsection{Frequency Change}

Consider an incompressible wave, either an Alfvén wave or a slow wave in the incompressible limit. Although the slow wave is, in fact, slightly compressible, this does not have a large effect on the final result. We calculate the frequency change of an incompressible wave with fixed $k: k_{\|}$ along the mean magnetic field and $k_{\perp}$ transverse to the mean magnetic field, where $k_{\|} \ll k_{\perp} \simeq k$.

We define $v$ to be the mean velocity of the protons averaged over the proton distribution function. Thus, $v$ satisfies the equation of motion for the Alfvén wave or for the slow wave derived in Appendix A. We then calculate the force that these protons exert on the neutrals. Since this force is the same as the force of the neutrals on the protons, inserting it into the equation of motion for the protons yields the change in frequency.

We denote the perturbed distribution function for the neutrals, i.e., perturbed to first order in $v_{N}$, after being Fourier transformed in space and time, by $\delta f_{N}\left(k, \omega, \boldsymbol{v}_{N}\right)$, where $v_{N}$ is the velocity of the neutrals. The evolution of the neutrals is determined by the Boltzmann equation,

$$
-i \omega \delta f_{N}+i\left(\boldsymbol{k} \cdot \boldsymbol{v}_{N}\right) \delta f_{N}=\mathscr{C},
$$

where $\mathscr{C}$ is the Fourier-transformed, perturbed, collision integral of the neutrals with the protons. Neutral-neutral 
collisions are negligible relative to neutral-proton collisions. The collision integral is simplified by making the approximation that, on the timescale that a neutral collides with protons, $\delta f_{N}$ is driven toward a Maxwellian with mean velocity $v$ :

$$
\mathscr{C}=v_{N, p}\left[\frac{\left(\boldsymbol{v} \cdot v_{N}\right)}{T / m_{N}} \frac{n_{N} \exp \left(-m_{N} v_{N}^{2} / 2 T\right)}{\left(2 \pi T / m_{N}\right)^{3 / 2}}-\delta f_{N}\right],
$$

where the first term in the square brackets is generated by expanding a Maxwellian distribution function with mean velocity $\boldsymbol{v}$ to linear order in $\boldsymbol{v}$ and retaining only the perturbation, and $v_{N, p}$ is the frequency with which a given neutral atom collides with protons. As is verified below, the perturbed neutral number density vanishes; therefore, it does not contribute to $\mathscr{C}$. With the above approximation to the collision integral, the solution to the Boltzmann equation reads

$$
\delta f_{N}=\frac{\left(\boldsymbol{v} \cdot v_{N}\right)}{T / m_{N}} \frac{n_{N} \exp \left(-m_{N} v_{N}^{2} / 2 T\right)}{\left(2 \pi T / m_{N}\right)^{3 / 2}} \frac{v_{N, p}}{v_{N, p}-i \omega+i \boldsymbol{k} \cdot v_{N}} .
$$

Next we verify that the perturbed neutral number density vanishes,

$$
\begin{aligned}
\int \delta f_{N} d^{3} v_{N}= & \frac{n_{N} v v_{N, p}}{(2 \pi)^{3 / 2}\left(T / m_{N}\right)^{5 / 2}} \\
& \times\left[\int d v_{N \|} v_{N \|} \exp \left(-\frac{m_{N} v_{N \|}^{2}}{2 T}\right)\right] \\
& \times\left[\int d^{2} v_{N \perp} \frac{\exp \left(-m_{N} v_{N \perp}^{2} / 2 T\right)}{v_{N, p}-i \omega+i k \cdot v_{N \perp}}\right] \\
= & 0,
\end{aligned}
$$

since the first square-bracketed integral vanishes by antisymmetry. For the purpose of evaluating the above integrals, we use $\perp$ and $\|$ for the perpendicular and parallel projections of $v_{N}$ relative to $v$, and not relative to the magnetic field as in the rest of this paper, i.e., $v_{N \|} \equiv\left(v \cdot v_{N}\right) v / v^{2}$ and $v_{N \perp} \equiv v_{N}-v_{N \|}$. We also use the incompressibility relation $\boldsymbol{k} \cdot \boldsymbol{v}=0$, which implies that $\boldsymbol{k} \cdot \boldsymbol{v}_{N \|}=0$.

With the neutral distribution function given by equation (24), the force per unit volume of the protons on the neutrals may be calculated as

$$
\begin{aligned}
\boldsymbol{F}= & m_{N} \int \boldsymbol{v}_{N}\left[-i \omega \delta f_{N}+i\left(\boldsymbol{k} \cdot \boldsymbol{v}_{N}\right) \delta f_{N}\right] d^{3} \boldsymbol{v}_{N} \\
= & \frac{m_{N} n_{N} \boldsymbol{v} v_{N, p}}{(2 \pi)^{3 / 2}\left(T / m_{N}\right)^{5 / 2}} \\
& \times\left[\int d v_{N \|} v_{N \|}^{2} \exp \left(-\frac{\left.\left.m_{N} v_{N \|}^{2}\right)\right]}{2 T}\right)\right] \\
& \times\left[\int d^{2} \boldsymbol{v}_{N \perp} \frac{\left(-i \omega+i \boldsymbol{k} \cdot \boldsymbol{v}_{N \perp}\right) \exp \left(-m_{N} v_{N \perp}^{2} / 2 T\right)}{v_{N, p}-i \omega+i \boldsymbol{k} \cdot \boldsymbol{v}_{N \perp}}\right] \\
= & \frac{m_{N} n_{N} v v_{N, p}}{\left(2 \pi T / m_{N}\right)^{1 / 2}} \\
& \times \int_{-\infty}^{\infty} d s \frac{(-i \omega+i k s) \exp \left(-m_{N} s^{2} / 2 T\right)}{v_{N, p}-i \omega+i k s} \\
= & \frac{m_{N} n_{N} v v_{N, p}}{\pi^{1 / 2} \Pi_{1}} \int_{-\infty}^{\infty} \frac{i t}{1+i t} \exp \left[-\left(\frac{t}{\Pi_{1}}+\frac{\Pi_{2}}{\Pi_{1}}\right)^{2}\right] d t .
\end{aligned}
$$

The second equality follows after replacing the overall multiplicative $v_{N}\left(=v_{N \|}+v_{N \perp}\right)$ by $v_{N \|}$ because the $v_{N \perp}$ term vanishes by antisymmetry of the integral over $v_{N \|}$. In the second equality, the first square-bracketed integral is equal to $(2 \pi)^{1 / 2}\left(T / m_{N}\right)^{3 / 2}$. The second square-bracketed integral is a double integral; the integral over the component of $v_{N \perp}$, that is perpendicular to $k$ is equal to $\left(2 \pi T / m_{N}\right)^{1 / 2}$. The remaining integral is over the component of $v_{N \perp}$, that is parallel to $k$; we label this component $s$ in the third equality. Finally, the fourth equality follows from the change of variables $t \equiv(k s-\omega) / v_{N, p}$ and from the following definitions:

$$
\begin{aligned}
& \Pi_{1} \equiv \frac{k}{v_{N, p}}\left(\frac{2 T}{m_{N}}\right)^{1 / 2}=\frac{v_{\mathrm{A}} k}{v_{N, p}} \beta^{1 / 2}\left(\frac{m_{p}}{m_{N}}\right)^{1 / 2}, \\
& \Pi_{2} \equiv \frac{\omega}{v_{N, p}}=\frac{v_{\mathrm{A}} k_{\|}}{v_{N, p}}
\end{aligned}
$$

where $m_{p}$ is the proton mass. The dimensionless parameter $\Pi_{1}$ is the number of wavelengths a neutral crosses before colliding, and the dimensionless parameter $\Pi_{2}$ is the number of wave periods a neutral travels before colliding. Since $k \gg k_{\|}$and $\beta \gtrsim 1, \Pi_{1} \gg \Pi_{2}$ in the inertial range of the MHD cascade.

Performing the integral for the real and imaginary parts of $\boldsymbol{F}$ to lowest order in $\Pi_{2} / \Pi_{1}$ yields

$$
\boldsymbol{F}=m_{N} n_{N} v v_{N, p}\left(1-2 i \frac{\Pi_{2}}{\Pi_{1}^{2}}\right) g\left(\Pi_{1}\right),
$$

where

$$
\begin{aligned}
g\left(\Pi_{1}\right) \equiv & 1-\frac{\sqrt{\pi} \exp \left(\Pi_{1}^{-2}\right) \operatorname{erfc}\left(\Pi_{1}^{-1}\right)}{\Pi_{1}} \\
& = \begin{cases}\frac{1}{2} \Pi_{1}^{2} & \text { for } \Pi_{1} \ll 1, \\
1 & \text { for } \Pi_{1} \gg 1 .\end{cases}
\end{aligned}
$$

Since $-\boldsymbol{F}$ is equal to the force of the neutrals on the protons, we insert $-\boldsymbol{F}$ into the equation of momentum conservation for the protons. More precisely, we add $-i \boldsymbol{F} /\left(m_{p} n\right)$ to the right-hand side of equation (A2) in Appendix A. We label the resulting change of frequency caused by the presence of neutrals as $\Delta \omega$. Assuming that $\Delta \omega \ll v_{\mathrm{A}} k_{\|}$, it follows that

$$
\frac{\Delta \omega}{v_{\mathrm{A}} k_{\|}}=-\frac{m_{N} n_{N}}{m_{p} n}\left(\frac{1}{\Pi_{1}^{2}}+\frac{i}{2 \Pi_{2}}\right) g\left(\Pi_{1}\right) .
$$

\subsubsection{Discussion of the Frequency Change}

The physical interpretation of this frequency change is straightforward. Recall that $k \gg k_{\|}$, so that the wavelength, $2 \pi / k$, is nearly identical to the wavelength transverse to the magnetic field, $2 \pi / k_{\perp}$. It is convenient to define the neutral mean free path, $L_{N}$, as follows:

$$
L_{N} \equiv \frac{\Pi_{1}}{k}=\frac{c_{T}}{v_{N, p}}\left(\frac{2 m_{p}}{m_{N}}\right)^{1 / 2},
$$

where $c_{T}=(p / \rho)^{1 / 2}$ is the isothermal sound speed. 
The imaginary part of $\Delta \omega$ is the negative of the damping rate and is given by

$$
\begin{aligned}
\omega_{i} & =-\frac{1}{2} \frac{m_{N} n_{N}}{m_{p} n} v_{N, p} g\left(k L_{N}\right) \\
& =-\frac{1}{2} \frac{m_{N} n_{N}}{m_{p} n} v_{N, p} \begin{cases}\frac{1}{2}\left(k L_{N}\right)^{2} & \text { for } k \mathrm{~L}_{N} \ll 1, \\
1 & \text { for } k \mathrm{~L}_{N} \gg 1 .\end{cases}
\end{aligned}
$$

The above expression may be explained as follows (Goldreich \& Sridhar 1995). The neutrals are nearly locked to the protons only if both $\Pi_{1}<1$ and $\Pi_{2}<1$. These conditions hold for wavelengths larger than the neutral mean free path $\left(k L_{N}<1\right)$. Thus, on these scales damping is due to the viscosity of the neutrals. For wavelengths smaller than the neutral mean free path, the neutral atoms are decoupled from the ions and hence are effectively at rest. The damping rate is thus the ion-neutral collision frequency.

The real part of the frequency change, $\Delta \omega_{r}$, is

$$
\frac{\Delta \omega_{r}}{v_{\mathrm{A}} k_{\|}}=-\frac{m_{N} n_{N}}{m_{p} n} \begin{cases}\frac{1}{2} & \text { for } k \mathrm{~L}_{N} \ll 1, \\ \left(k L_{N}\right)^{-2} & \text { for } k \mathrm{~L}_{N} \gg 1 .\end{cases}
$$

Its physical significance is apparent. For small wavelengths the neutral atoms are effectively freely streaming, the motion of the protons nearly decouples from the motion of the neutrals, and there is negligible real frequency change associated with the presence of the neutrals. For large wavelengths the neutrals are locked to the protons, so the mass density of the fluid that participates in the waves is larger than that of the protons. Since the Alfvén speed, and hence the wave frequency, is inversely proportional to the square root of the mass density, i.e., $\omega \propto v_{\mathrm{A}} \propto \rho^{-1 / 2}$, this results in a decrease of the frequency by a fractional amount that is equal to one-half of the mass ratio of neutrals to protons.

\subsection{Effect of Neutrals on the Turbulent Cascade \\ 6.2.1. Effect of $\omega_{i}$ on the Cascade}

The turbulent cascade is quenched if the damping rate exceeds the eddy cascade rate or, equivalently, if $\left|\omega_{i}\right| / v_{\mathrm{A}} k_{\|}>1$. From equation (39) and $k_{\|} \propto k^{2 / 3}$, it is seen that for small wavenumbers $\left|\omega_{i}\right| / v_{\mathrm{A}} k_{\|} \propto k^{4 / 3}$ and for large wavenumbers $\left|\omega_{i}\right| / v_{\mathrm{A}} k_{\|} \propto k^{-2 / 3}$. Therefore, $\left|\omega_{i}\right| / v_{\mathrm{A}} k_{\|}$is a maximum for transverse wavelengths, $k^{-1}$, comparable to the neutral mean free path, and decreases for both larger and smaller wavelengths. The requirement that the cascade survive damping by neutrals is then that $\left|\omega_{i}\right| / v_{\mathrm{A}} k_{\|}<1$ for $k^{-1}$ comparable to the neutral mean free path, or equivalently, $n_{N} v_{N, p} / n \lesssim v_{\mathrm{A}} k_{\|}$at this scale (eq. [39]). Since $n_{N} v_{N, p} / n$ is the rate at which a given proton collides with neutrals, the cascade survives to small scales if the wave frequency, and hence the cascade rate, at the scale of the neutral mean free path is faster than the rate at which a proton collides with neutrals.

To obtain the total decrement in the amplitude of both the Alfvén mode and the slow mode through the damping scale, we solve the kinetic equation for the cascade. ${ }^{8}$ As in $\S$ 5.1.1, the kinetic equation is obtained by balancing the $k$-space energy flux with the loss rate of $k$-space energy density due to damping:

$$
\frac{d}{d k} \frac{v_{\lambda_{\perp}}^{2}}{t_{\lambda_{\perp}}}=2 \omega_{i} \frac{v_{\lambda_{\perp}}^{2}}{k}
$$

where $\lambda_{\perp}^{-1} \equiv k_{\perp} \simeq k$ is the transverse wavenumber and $t_{\lambda_{\perp}}$ is the cascade time. The kinetic equation can be rewritten as follows:

$$
\frac{d}{d \ln k} \ln \frac{v_{\lambda_{\perp}}^{2}}{t_{\lambda_{\perp}}}=2 M_{t} \frac{\omega_{i}}{v_{\mathrm{A}} k_{\|}},
$$

where $M_{t}$ is a Kolmogorov constant (see $\S 2.5 .1$ ). Before integrating this equation, we reexpress the damping frequency in terms of the relevant length scales, $L_{\text {out }}$ and $L_{N}$ :

$$
\begin{aligned}
\frac{\omega_{i}}{v_{\mathrm{A}} k_{\|}} & =-\frac{1}{2} \frac{m_{N} n_{N}}{m_{p} n} v_{N, p} \frac{g\left(k L_{N}\right)}{v_{\mathrm{A}} k_{\|}} \\
& =-\frac{1}{2 M_{\|}} \frac{m_{N} n_{N}}{m_{p} n} v_{N, p} \frac{g\left(k L_{N}\right)}{v_{\lambda_{\perp}} k} \\
& =-\frac{1}{\sqrt{2} M_{\|}}\left(\frac{m_{N}}{m_{p}}\right)^{1 / 2} \frac{n_{N}}{n}\left(\frac{L_{\text {out }}}{L_{N}}\right)^{1 / 3} \frac{g\left(k L_{N}\right)}{\left(k L_{N}\right)^{2 / 3}} .
\end{aligned}
$$

The first equality above follows from equation (38). The second equality follows from the Kolmogorov constant $M_{\|}=v_{\mathrm{A}} k_{\|} / v_{\lambda_{\perp}} k$ (see $\left.\S 2.5 .1\right)$. The third equality follows from the velocity spectrum, $v_{\lambda_{\perp}}=c_{T}\left(k L_{\text {out }}\right)^{-1 / 3}$ (eq. [12]), and from the definition of the neutral mean free path (eq. [37]). For convenience, we define $L_{\text {out }}$ in this section such that $v_{\lambda_{\perp}}=c_{T}$ when $k^{-1}=L_{\text {out }}$.

Integrating the kinetic equation (eq. [42]) and using $t_{\lambda_{\perp}} \sim \lambda_{\perp} / v_{\lambda_{\perp}}$ yields the net decrement due to damping by collisions with neutrals:

$$
\begin{aligned}
\frac{\left.\left(v_{\lambda_{\perp}} / \lambda_{\perp}^{1 / 3}\right)\right|_{\lambda_{\perp} \ll L_{N}}}{\left.\left(v_{\lambda_{\perp}} / \lambda_{\perp}^{1 / 3}\right)\right|_{\lambda_{\perp} \gg L_{N}}} & =\exp \left(\frac{2 M_{t}}{3} \int_{0}^{\infty} \frac{\omega_{i}}{v_{\mathrm{A}} k_{\|}} \frac{d k}{k}\right) \\
& =\exp \left[-0.2\left(\frac{m_{N}}{m_{p}}\right)^{1 / 2} \frac{n_{N}}{n}\left(\frac{L_{\text {out }}}{L_{N}}\right)^{1 / 3}\right],
\end{aligned}
$$

after substituting from equation (45). The numerical prefactor in the second equality, 0.2 , follows after inserting the values of the Kolmogorov constants (eq. [8]) and the value of the integral

$$
\int_{0}^{\infty} g(x) x^{-5 / 3} d x \simeq 1.1
$$

which was integrated numerically; see equation (34) for the definition of $g(x)$.

For the cascade to continue to small scales, the righthand side of equation (46) cannot be very small. This can be viewed as an upper limit on the neutral fraction: $n_{N} / n \lesssim$ $\left(L_{N} / L_{\text {out }}\right)^{1 / 3}$. Recall that the condition for the cascade to reach small scales is that the cascade rate at $L_{N}$ be faster

\footnotetext{
${ }^{8}$ The calculation of the amplitude decrement in this section is similar to that given in $\S 5.1 .1$ for slow mode damping at the cooling scale. The principal difference, aside from the fact that the imaginary part of the frequency is different, is that here we must account for the decrease in the cascade rate, $t_{\lambda_{1}}$, caused by damping. This is not necessary when treating slow mode damping at the cooling scale because there the Alfvén modes that control the cascade rate are undamped.
} 
than the rate at which a proton collides with neutrals. If the neutral fraction is too large, then so is the proton collision rate, and the cascade is quenched. Moreover, for a fixed $L_{N}$, a large value for $L_{\text {out }}$ implies that the cascade time at $L_{N}$ is large and hence that the cascade is more susceptible to damping by neutrals.

We postpone consideration of the damping due to hydrogen and helium atoms until after we evaluate the effect of $\Delta \omega_{r}$ on the cascade.

\subsubsection{Effect of $\Delta \omega_{r}$ on the Cascade}

We can picture the cascade as proceeding from large scales to small scales. As it crosses the scale of the neutral mean free path (eq. [40]), the effective Alfvén speed, and hence the real part of wave frequency, increases by the fraction $m_{N} n_{N} / 2 m_{p} n$. Consequently, the cascade time decreases by the same amount. To the extent that the flux of energy in Alfvén waves from large scales to small scales is constant in the turbulent cascade, $v_{\lambda_{\perp}}^{2} / t_{\lambda_{\perp}}=$ constant, where $t_{\lambda_{\perp}}$ is the cascade time. Therefore, a fractional decrease of $m_{N} n_{N} / 2 m_{p} n$ in the cascade time causes a decrement in the small-scale Alfvénic velocity perturbation by the fractional amount $m_{N} n_{N} / 4 m_{p} n$. This decrement is in addition to that due to damping. Moreover, it applies to the slow mode and the entropy mode as well as to the Alfvén mode. Because $n_{N} / n$ must be small for the cascade to pass through the scale of neutral damping, this decrement is also small, and we ignore it from here on.

\subsection{Neutral Hydrogen Atoms}

When the neutrals are hydrogen atoms, we set $n_{N}=n_{\mathrm{H}}$, $v_{N, p}=v_{\mathrm{H}, p}, L_{N}=L_{\mathrm{H}}$, and $m_{N}=m_{p}$. Collisions between hydrogen atoms and protons are due to resonant charge exchange. From equation (37), with the value of $v_{\mathbf{H}, p}$ taken from Banks (1966),

$$
L_{\mathrm{H}}=5 \times 10^{13}\left(\frac{\mathrm{cm}^{-3}}{n}\right) \mathrm{cm}
$$

at $8000 \mathrm{~K}$; the temperature dependence of $L_{\mathrm{H}}$ is very weak.

For the cascade to survive damping by neutral hydrogen atoms, the right-hand side of equation (46) cannot be very small. This sets an upper limit on the neutral fraction of

$$
\frac{n_{\mathrm{H}}}{n} \lesssim 5\left(\frac{L_{\mathrm{H}}}{L_{\text {out }}}\right)^{1 / 3} \sim\left[\frac{6 \times 10^{15} \mathrm{~cm}}{\left(n / \mathrm{cm}^{-3}\right) L_{\text {out }}}\right]^{1 / 3} .
$$

The real part of $\omega / v_{\mathrm{A}} k_{\|}$is larger on scales below $L_{\mathrm{H}}$ than it is on scales above $L_{\mathrm{H}}$ by the fractional value $n_{\mathrm{H}} / 2 n$.

\subsection{Neutral Helium Atoms}

Although helium has a lower abundance than hydrogen, it has a higher ionization potential. Therefore, in some regions helium might comprise the majority of neutrals. When the neutrals are helium atoms, we set $n_{N}=n_{\mathrm{He}}$, $v_{N, p}=v_{\mathrm{He}, p}, L_{N}=L_{\mathrm{He}}$, and $m_{N}=4 m_{p}$. From equation (37), with the value of $v_{\mathrm{He}, p}$ taken from Banks (1966),

$$
L_{\mathrm{He}}=1.5 \times 10^{15}\left(\frac{\mathrm{cm}^{-3}}{n}\right) \mathrm{cm}
$$

at $8000 \mathrm{~K}$ and $L_{\mathrm{He}} \propto T^{1 / 2}$.

To place an upper bound on damping by helium atoms, we assume that most of the helium is neutral, i.e., $n_{\mathrm{He}} \approx 0.1 n$. Then the decrement is given by the right-hand side of equation (46):

$$
\frac{\left.\left(v_{\lambda_{\perp}} / \lambda_{\perp}^{1 / 3}\right)\right|_{\lambda_{\perp} \ll L_{\mathrm{He}}}}{\left.\left(v_{\lambda_{\perp}} / \lambda_{\perp}^{1 / 3}\right)\right|_{\lambda_{\perp} \gg L_{\mathrm{He}}}}=\exp \left\{-\left[\frac{\left(n / \mathrm{cm}^{-3}\right) L_{\mathrm{out}}}{2 \times 10^{19} \mathrm{~cm}}\right]^{1 / 3}\right\} .
$$

Only if $L_{\text {out }} \gtrsim 2 \times 10^{19}\left(\mathrm{~cm}^{-3} / n\right) \mathrm{cm}$ could the cascade be terminated at the scale of the helium mean free path. If there are not many neutral helium atoms, or if the outer scale is not sufficiently large, then damping at $L_{\mathrm{He}}$ may be neglected, and the cascade extends at least to the scale of the hydrogen mean free path.

Collisions of neutral helium atoms with singly ionized helium ions might also be significant. Although there are fewer helium atoms than protons, $\mathrm{He}^{0}-\mathrm{He}^{+}$collisions have a larger cross section than $\mathrm{He}^{0}$-proton collisions because they are due to resonant charge exchange. Nonetheless, given the cosmic abundance of helium, the mean free path of neutral helium due to $\mathrm{He}^{0}-\mathrm{He}^{+}$collisions cannot be significantly smaller than that due to $\mathrm{He}^{0}$-proton collisions regardless of the ionization fraction of helium.

The real part of $\omega / v_{\mathrm{A}} k_{\|}$increases by $2 n_{\mathrm{He}} / n$ below the scale $L_{\mathrm{He}}$, or by 0.2 if most of the helium is neutral.

\subsection{If Neutrals Damp the Cascade}

In subsequent sections we consider regions in the interstellar medium where there are too few neutrals to damp the cascade. However, there are almost certainly many regions where the neutrals do damp the cascade. We discuss these regions here.

Suppose both Alfvén waves and slow waves are damped by neutrals. What happens to the entropy waves? If undamped, they would be mixed down to smaller scales by Alfvén waves at the neutral damping scale. The resulting density spectrum would be $n_{\lambda_{\perp}}=$ constant (see eq. [5]). Because it is flatter than $n_{\lambda_{\perp}} \propto \lambda_{\perp}^{1 / 3}$, regions in which the Alfvén cascade is truncated by damping by neutrals might be important contributors to small-scale density fluctuations. However, the fact that they would contribute a density spectrum different from that which is observed suggests that the entropy wave cascade is not more resistant than the Alfvén wave cascade to damping by neutrals. Indeed that is the case. Recall that the condition for the truncation of Alfvén and slow wave cascades is that each proton collide with at least one neutral atom during one wave period at the neutral damping scale. Under this condition, the neutrals would damp the entropy waves by conducting heat across them.

Although regions in which the cascade is damped by neutrals do not contribute small-scale density fluctuations, they may still be significant. Observational evidence indicates that there is more power in density fluctuations on large scales, $10^{13}-10^{14} \mathrm{~cm}$, than would be predicted by extrapolating from small scales, $10^{8}-10^{10} \mathrm{~cm}$, with the Kolmogorov scaling. See, for example, Lambert \& Rickett (2000) for a review of the observations. Perhaps this excess arises in regions where the cascade is damped by neutrals.

We complete this section by briefly considering and then rejecting the possibility that a turbulent cascade truncated at the neutral damping scale might be regenerated on a much smaller scale as a result of stirring by eddies at the damping scale. Although the ratio of the damping rate to the wave frequency decreases below the scale of the neutral mean free path, the absolute damping rate approaches a constant value. Provided that the cascade is truncated by 
neutral damping, this rate is larger than the stirring rate and the cascade cannot be regenerated.

\section{THE COLLISIONLESS SCALE OF THE IONS}

If the cascade survives the neutral collisionless scales, then, proceeding to smaller scales, the next scale of importance is the ion collisionless scale. This scale is set by the mean free path of protons to collide with other protons:

$$
L_{\mathrm{mfp}}=6 \times 10^{11}\left(\frac{\mathrm{cm}^{-3}}{n}\right) \mathrm{cm}
$$

at a temperature of $8000 \mathrm{~K}$ (Braginskii 1965). Since the electron and proton gyroradii (see eq. [62] for protons) are very small compared to the proton mean free path, the electrons and protons are tied to magnetic field lines. Therefore, when considering the collisionless effects of electrons and protons, the relevant length scales are those parallel to the magnetic field. Since turbulent eddies are highly elongated along the magnetic field, their transverse length scales are much smaller than their parallel ones (eq. [2]). It is the transverse length scale that is relevant when considering observations of the density spectrum because each line of sight averages over regions with different magnetic field orientations. In this section we show that both the slow mode and the entropy mode are cut off at the length scale where the parallel eddy size is comparable to the proton mean free path. The length scale where the density spectrum is observed to cut off, i.e., the transverse length scale, is therefore significantly smaller than the mean free path.

Before discussing the damping of the slow mode and the entropy mode, we consider two larger length scales, which are set by the effects of the electrons. Although the behavior of the cascade at these length scales is interesting, it is shown to be unimportant for our purposes.

Throughout this section we neglect numerical factors of order unity, such as the factors that are associated with kinetic corrections to the fluid equations (given in Braginskii 1965) and the Kolmogorov constants. However, we retain the dependences on $\beta$, which is assumed to satisfy $\beta>1$, and on the ratio of the proton mass to the electron mass, $m_{p} / m_{e}=1840$.

\subsection{The Electron Diffusion Scale}

The electrons have the same mean free path as the protons, but they are faster than the protons by the square root of the mass ratio:

$$
c_{s, e} \approx\left(\frac{m_{p}}{m_{e}}\right)^{1 / 2} c_{s}
$$

where $c_{s, e}$ is the electron thermal speed and $c_{s}$ is the sound speed, which is comparable to the proton thermal speed. Because of charge neutrality, the electrons have the same density, both perturbed and unperturbed, as the protons. Viscous damping caused by the electrons may be neglected: since the dynamic viscosity of the electrons is smaller than that of the protons by the square root of their mass ratio, electron viscous damping is always subdominant.

As we now show, electrons are important for conducting heat on parallel length scales slightly larger than the proton mean free path. Electrons diffuse parallel to the magnetic field across a distance $\Lambda_{\|}$in the time $\left(L_{\mathrm{mfp}} / c_{s, e}\right)\left(\Lambda_{\|} / L_{\mathrm{mfp}}\right)^{2}$. This is equal to the cascade time of an eddy with parallel length scale $\Lambda_{\|}$, i.e., it is equal to $\Lambda_{\|} / v_{\mathrm{A}}$, at the "electron diffusion scale," given by $\Lambda_{\|} \sim L_{\text {ed }}$, where

$$
L_{\mathrm{ed}} \approx \frac{c_{s, e}}{v_{\mathrm{A}}} L_{\mathrm{mfp}} \approx \beta^{1 / 2}\left(\frac{m_{p}}{m_{e}}\right)^{1 / 2} L_{\mathrm{mfp}} .
$$

In eddies with parallel lengths smaller than this, the electrons diffuse across the parallel lengths of the eddies, thereby conducting heat, and the electrons are isothermal; in eddies with parallel lengths larger than this, conduction is unimportant.

We must also consider the effect of electron conduction on the protons. Electron and proton temperatures approach a common value on the timescale that there are $m_{p} / m_{e}$ collisions per particle. Since electrons are faster, the collision time is set by the electron speed, and the time for electron and proton temperatures to equilibrate is

$$
\tau_{\mathrm{eq}} \approx \frac{m_{p}}{m_{e}} \frac{L_{\mathrm{mfp}}}{c_{s, e}} \approx \frac{1}{v_{\mathrm{A}}} \frac{1}{\beta^{1 / 2}}\left(\frac{m_{p}}{m_{e}}\right)^{1 / 2} L_{\mathrm{mf} p} .
$$

This time is smaller, by a factor $\beta$, than the cascade time of eddies with parallel size $L_{\text {ed }}$. Thus, in eddies of this parallel size, and in those that are slightly smaller, protons are at the same temperature as the electrons, and since the electrons are isothermal, so are the protons.

As a result of the above considerations, when the parallel cascade crosses the electron diffusion scale, the cascade becomes isothermal. This is similar to the crossing of the cooling scale, discussed in $\S 5.1$, though backward, and similar considerations apply. In particular, the Alfvén mode is incompressible and hence unaffected. The slow mode is nearly incompressible and is only slightly affected: it suffers some damping to first order in $1 / \beta$. The entropy mode, however, is rapidly damped under isothermal conditions; as the electron diffusion scale is crossed, entropy waves are converted into slow waves, and the density fluctuations that had been associated with entropy waves now become associated with slow waves.

\subsection{The Electron-Proton Equilibration Scale}

Continuing to slightly smaller scales, the isothermal cascade reaches the "equilibration scale," where the cascade time, $\Lambda_{\|} / v_{\mathrm{A}}$, is comparable to the electron-proton equilibration time (eq. [55]). At this scale, $\Lambda_{\|} \sim L_{\text {eq }}$, where

$$
L_{\mathrm{eq}} \approx \frac{1}{\beta^{1 / 2}}\left(\frac{m_{p}}{m_{e}}\right)^{1 / 2} L_{\mathrm{mfp}} .
$$

On larger scales, the protons are thermally coupled to the electrons, and hence they are isothermal on slightly larger scales; on smaller scales, the protons are thermally independent of the electrons and hence adiabatic. The transition through the equilibration scale is nearly identical to the transition through the cooling scale (§ 5.1): the Alfvén waves are unaffected, and they mix larger scale isothermal slow waves into smaller scale adiabatic slow waves and into smaller scale entropy waves, although with some damping. Therefore, below the equilibration scale, the entropy mode reappears.

The result of the calculations in both this subsection and the previous subsection is that, in crossing the electron diffusion scale and the equilibration scale, density fluctuations that were associated with the entropy mode on large scales are transferred from the entropy mode to the slow mode and then back to the entropy mode. Density fluctuations 
that were associated with the slow mode on large scales are unaffected. ${ }^{9}$ Therefore, for our purposes, these two length scales have little net effect on the density spectrum. Although there is some damping, the amount of damping is comparable to the amount at the cooling scale and hence is not very significant.

\subsection{The Proton Diffusion Scale: Death of the Slow Mode and Entropy Mode}

Entropy waves and slow waves with parallel wavelengths smaller than the proton mean free path both damp on the timescale that protons stream across their parallel wavelengths (Barnes 1966). However, because we consider $\beta>1$, there is a scale slightly larger than the proton mean free path at which these waves damp in the turbulent cascade. This is the proton diffusion scale, which is the scale at which protons can diffuse across an eddy within a cascade time. Below the proton diffusion scale, proton viscosity kills the slow waves and proton heat conduction kills the entropy waves. Alfvén waves are unaffected by either of these effects. The density spectrum therefore cuts off below the proton diffusion scale.

To evaluate the proton diffusion scale, we equate the time for protons to diffuse across an eddy of parallel length scale $\Lambda_{\|}$, i.e., $\left(L_{\mathrm{mfp}} / c_{s}\right)\left(\Lambda_{\|} / L_{\mathrm{mfp}}\right)^{2}$, with the cascade time, $\Lambda_{\|} / v_{\mathrm{A}}$. This gives $\Lambda_{\|} \sim L_{\mathrm{pd}}$, where

$$
L_{\mathrm{pd}} \approx \frac{c_{s}}{v_{\mathrm{A}}} L_{\mathrm{mfp}} \approx \beta^{1 / 2} L_{\mathrm{mfp}}
$$

However, from an observational point of view, it is the transverse size of a damped eddy, $\lambda_{\perp}$, that would be observed. This is related to the parallel size through equation (2). Alternatively, we use the Kolmogorov constant $M_{\|} \equiv v_{\mathrm{A}} \lambda_{\perp} / v_{\lambda_{\perp}} \Lambda_{\|}($see $\S 2.5 .1) .{ }^{10}$ Then, the transverse scale of a damped eddy, i.e., an eddy with parallel size $\Lambda_{\|} \sim L_{\mathrm{pd}}$, is

$$
\begin{aligned}
\lambda_{\perp} & \approx M_{\|} \frac{v_{\lambda_{\perp}}}{v_{\mathrm{A}}} L_{\mathrm{pd}} \\
& \approx M_{\|} \beta L_{\mathrm{mfp}}\left(\frac{\lambda_{\perp}}{L_{\mathrm{out}}}\right)^{1 / 3},
\end{aligned}
$$

which we solve for the cutoff length scale: $\lambda_{\perp} \sim L_{\mathbf{p d}}^{(\perp)}$, where

$$
\begin{aligned}
L_{\mathrm{pd}}^{(\perp)} & \approx M_{\|}^{3 / 2} \beta^{3 / 2} L_{\mathrm{mfp}}\left(\frac{L_{\mathrm{mfp}}}{L_{\mathrm{out}}}\right)^{1 / 2} \\
& \approx 2 \times 10^{9}\left(\frac{\mathrm{pc}}{L_{\text {out }}}\right)^{1 / 2}\left(\frac{\beta}{n / \mathrm{cm}^{-3}}\right)^{3 / 2} \mathrm{~cm} .
\end{aligned}
$$

Below this scale, the density spectrum is cut off. For plausible values of $\beta, L_{\text {out }}$, and $n$, this length scale is significantly

\footnotetext{
${ }^{9}$ However, if the density fluctuations in the large-scale slow mode were less than the density fluctuations in the large-scale entropy mode, then, on small scales, the slow mode would be boosted so that its density fluctuations would be comparable to those of the entropy mode: the slow mode can steal approximately half of the entropy mode. Note that, since the behavior of the cascade at the electron-proton equilibration scale is nearly identical to the behavior at the cooling scale, similar uncertainties apply; see discussion in $\S 5.1$. In particular, we are unable to quantify the damping of the entropy fluctuations, although we expect that this damping is not so large as to render the entropy mode negligible relative to the slow mode on small scales.

${ }^{10}$ Although we drop other factors of order unity, we keep the dependence on this one because of the importance of the proton diffusion scale.
}

smaller than $L_{\mathrm{mfp}}$. It is also larger than the proton gyroradius (eq. [62]).

Armstrong et al. (1995) summarize the observations of the density spectrum cutoff. There is considerable evidence that the cutoff scale is smaller than about $10^{10} \mathrm{~cm}$ along many directions. There is weaker evidence, from refractive scintillation, that along some lines of sight the cutoff scale is larger than around $10^{9} \mathrm{~cm}$. Our theory might have implications for these observations.

We conclude this section with two remarks. First, we reemphasize the importance of the fact that the cascade is anisotropic. It is this fact that allows the density spectrum to reach length scales that are significantly smaller than the proton mean free path. Second, we note an important consequence of the parallel cascades of the slow mode and of the passive scalar; these parallel cascades were explained in great detail when considering incompressible MHD turbulence $(\S 2)$. Had there been no parallel cascade, then neither the slow mode nor the entropy mode would damp at the proton diffusion scale. The wavelength along the magnetic field would be effectively infinite, and so the ions could not diffuse across wavelengths. Rather, the slow mode and the entropy mode would be mixed down to the proton gyroscale, where the Alfvénic cascade is cut off.

\subsection{Density Spectrum below the Proton Diffusion Scale}

On scales smaller than $L_{\text {pd }}^{(\perp)}$, density fluctuations are wiped out. Since on these scales protons diffuse across the lengths of many eddies before the eddies cascade, the density within neighboring eddies is homogenized. Homogenization occurs not only in the direction parallel to the local mean magnetic field (as might have been expected since protons are tied to field lines) but also in the transverse direction. This is because eddies that are adjacent in the parallel direction incorporate substantially different field lines, so proton diffusion also wipes out density differences among eddies with transverse separations. The result is that the density spectrum on scales smaller than $L_{\mathbf{p d}}^{(\perp)}$ is determined by density fluctuations at $L_{\mathbf{p d}}^{(\perp)}$; equivalently,

$$
n_{\lambda_{\perp}} \propto \lambda_{\perp}, \text { for } \lambda_{\perp}<L_{\mathbf{p d}}^{(\perp)} \text {. }
$$

\section{THE END: THE PROTON GYROSCALE}

The Alfvén mode is undamped in a collisionless medium (Barnes 1966). Thus, the Alfvén wave cascade survives below the proton diffusion scale, without the accompaniment of the slow waves and entropy waves. The Alfvénic cascade is damped at the scale of the proton gyroradius:

$$
L_{p, \mathrm{gyr}}=\sqrt{2} c_{T} \frac{m_{p} c}{e B}=2.5 \times 10^{7}\left(\frac{\beta}{n / \mathrm{cm}^{-3}}\right)^{1 / 2} \mathrm{~cm} .
$$

At this scale, Alfvén waves are converted into whistlers. The whistlers cascade to smaller scales, where they are damped by the collisionless effects of the electrons (Quataert 1998, viz., the curve in his Fig. $1 b$ that corresponds to equal electron and proton temperatures).

\section{SUMMARY}

The primary goal of this paper has been to calculate the small-scale density spectrum in turbulent interstellar plasmas. Our theory of compressible MHD turbulence is 
based upon the incompressible theory of Goldreich \& Sridhar (1995), for which there is growing support from numerical simulations. We hypothesize that the compressible theory is similar to the incompressible theory of MHD turbulence, but with two main modifications: a compressible slow mode and an entropy mode that is passively advected. While we believe that this hypothesis is plausible, it can, and should, be tested with numerical simulations of compressible MHD.

Because of the multitude of special length scales encountered when discussing the turbulent cascade in the interstellar medium, a recapitulation might be useful. In the following, we list the most important length scales and summarize their significance to the turbulent cascade.

The outer scale $\left(L_{\mathrm{out}}\right)$.- - This is the length scale at which the turbulent motions are stirred. On slightly smaller scales, there is isothermal hydrodynamic turbulence for most plausible astrophysical sources.

The MHD scale $\left(L_{\mathrm{MHD}}\right)$.- At this length scale there is a transition from hydrodynamic to MHD turbulence. Larger scale hydrodynamic motions couple to smaller scale Alfvén waves and slow waves.

The cooling scale $\left(L_{\mathrm{cool}}\right)$.- At this length scale there is a transition from isothermal to adiabatic turbulence. In high- $\beta$ turbulence, where small-scale density fluctuations are due to the entropy mode, entropy (and hence density) fluctuations are suppressed by cooling. In low- $\beta$ turbulence, i.e., $1 \lesssim \beta<L_{\text {out }} / c_{s} t_{\text {cool }}$, density fluctuations due to the slow mode are important, and cooling has a negligible effect on the small-scale density spectrum.

The collisionless scale of the neutrals $\left(L_{\mathrm{H}} ; L_{\mathrm{He}}\right)$. - Neutrals decouple from ions across these scales. If the neutral fraction is not sufficiently small, then all three modes (Alfvén, slow, and entropy) are damped.

The collisionless scale of the ions $\left(\Lambda_{\|}=L_{\mathrm{ed}} ; \Lambda_{\|}=L_{\mathrm{eq}}\right.$; $\left.\Lambda_{\|}=L_{\mathrm{pd}} \Leftrightarrow \lambda_{\perp}=L_{\mathrm{pd}}^{(\perp)}\right)$. - Across these length scales there is a gradual transition from fluid behavior to collisionless plasma behavior. The first two of these length scales, the electron diffusion scale and the equilibration scale, are set by the electrons. These length scales have only a small net effect on the cascade. The proton diffusion scale, however, is critically important for density fluctuations: both the slow mode and the entropy mode are cut off at this scale, and hence there are no density fluctuations below $L_{\mathrm{pd}}^{(\perp)}$.

The proton gyroscale $\left(L_{p, \mathrm{gyr}}\right)$.- -Alfvén waves are cut off at this scale. However, this scale has little importance for the density spectrum because there are no density fluctuations below the proton diffusion scale.

\section{COMPARISON WITH HIGDON'S WORK}

In his 1984 paper, Higdon attributes small-scale density fluctuations to the passive mixing of the entropy mode. In his 1986 paper, Higdon notes that if the entropy mode varies along the magnetic field, it is damped in a collisionless medium. He then attributes small-scale density fluctuations to the passive mixing of tangential pressure balances. Tangential pressure balances are structures that are parallel to the mean magnetic field. They are composed of both entropy waves and slow waves that have purely transverse wavevectors.

Considering that Higdon's papers preceded even a theory of incompressible MHD turbulence, they are a remarkable accomplishment. However, Higdon does not account for the parallel cascade. Entropy and slow waves with purely transverse wavevectors contain negligible power. They are cascaded along the magnetic field by Alfvén waves. Consequently, entropy and slow waves are damped when protons can diffuse across eddies in a cascade time, i.e., below the proton diffusion scale.

\section{COMPRESSIBLE TURBULENCE WHEN $\beta<1$}

In this section only, we consider compressible turbulence in plasmas that have $\beta<1$, e.g., in strong oblique isothermal shocks and in the solar wind.

Since Alfvén waves are unaffected by the value of $\beta$, and since nearly transverse slow waves are only slightly affected (see Appendix A), the dynamics of the cascade is nearly independent of $\beta$. While the dispersion relation of the slow mode is changed from $\omega=v_{\mathrm{A}} k_{z}$ to $\omega=c_{s} k_{z}$, the slow waves are still passively mixed by the Alfvén waves. Therefore, the Alfvén, slow, and entropy spectra in $\beta<1$ MHD turbulence are the same as when $\beta>1$.

However, the damping of the slow and entropy waves is significantly changed. When $\beta>1$, the proton thermal speed is faster than the Alfvén speed. Therefore, protons can stream across small eddies before they cascade. Conversely, when $\beta<1$, the time for protons to cross an eddy is always shorter than the cascade time. Therefore, slow and entropy waves cannot be damped by protons that cross eddies, and the density spectrum extends to smaller scales.

We have ignored the effects of the electrons. However, in the following we show that the electrons' behavior may be ignored for our purposes. Our discussion closely parallels that when $\beta>1$ in $\S 7$ and uses similar notation. When $\beta<1$, the equilibration scale (eq. [56]) is larger than the electron diffusion scale (eq. [54]). Thus, the largest scale at which kinetic effects are significant is the scale at which the cascade time is comparable to the time for electrons and protons to equilibrate their temperatures. Below this scale, electrons and protons are thermally decoupled. Nonetheless, this thermal decoupling has no effect on the Alfvén, slow, or entropy waves on scales larger than the electron diffusion scale. At the electron diffusion scale, the cascade time is comparable to the electron diffusion time. Below this scale, the electrons are isothermal. However, since the protons are thermally decoupled from the electrons, the slow waves and entropy waves are cascaded to smaller scales. $^{11}$

Continuing to smaller scales, the next scale of importance is that at which the parallel size of an eddy is comparable to the proton mean free path. Below this scale, the cascade is collisionless. The Alfvén waves are undamped by collisionless effects. The entropy waves are undamped since protons cannot cross eddies within a cascade time. The slow waves are also undamped within a cascade time: although they damp within a wave period by Barnes damping, their wave period is longer than the cascade time. Consequently, the Kolmogorov density spectrum extends down to the proton gyroscale.

\section{FUTURE WORK}

In a future paper we will examine in detail the density spectrum in the solar wind. In a follow-up paper we will relate the theory of compressible plasma turbulence developed here to observations, primarily those of diffractive

\footnotetext{
${ }^{11}$ There is a change in the density spectrum at this scale that is of order unity; it is due to the change in the electrons' equation of state.
} 
scintillation. We will demonstrate that the observed amplitude of small-scale density fluctuations is surprisingly large, especially along certain lines of sight, such as the one toward the Galactic center. Then we will attempt to determine which astrophysical sources contribute the bulk of the density fluctuations and why they do so.
We thank Jason Maron for analyzing some of his simulations for us, for showing us how to use his numerical code, and for informative discussions. We thank the referee, Ben Chandran, for a very helpful report. Research reported in this paper was supported by NSF grant 94-14232.

\section{APPENDIX A}

\section{WAVES IN COMPRESSIBLE MHD}

In this appendix we derive the properties of the Alfvén mode, the slow mode, and the entropy mode. The fast mode is not relevant to interstellar scintillation for reasons discussed in the body of the paper. The Fourier-transformed, linearized equations of ideal MHD, with $\partial / \partial t \rightarrow-i \omega$ and $\boldsymbol{\nabla} \rightarrow i k$, read

$$
\begin{aligned}
& \omega n^{\prime}=(\boldsymbol{k} \cdot \boldsymbol{v}) n \quad(\text { mass conservation), } \\
& \omega \boldsymbol{v}=\boldsymbol{k}\left(\tilde{c}^{2} \frac{n^{\prime}}{n}+b_{z} v_{\mathrm{A}}\right)-\boldsymbol{b} v_{\mathrm{A}} k_{z} \quad \text { (momentum conservation), } \\
& \omega \boldsymbol{b}=\hat{z} v_{A}(\boldsymbol{k} \cdot \boldsymbol{v})-\boldsymbol{v} v_{\mathrm{A}} k_{z} \quad \text { (Faraday's law), }
\end{aligned}
$$

where $n$ is number density, $\rho$ is the mass density, $B$ is the background magnetic field intensity, and $v_{\mathrm{A}} \equiv B /(4 \pi \rho)^{1 / 2}$ is the Alfvén speed. The $z$-axis is chosen to lie parallel to the background magnetic field. Some other variables are the perturbed number density, $n^{\prime}$; the fluid velocity, $\boldsymbol{v}$; and the perturbed magnetic intensity, $\boldsymbol{B}^{\prime}$, or in velocity units, $\boldsymbol{b} \equiv \boldsymbol{B}^{\prime} /(4 \pi \rho)^{1 / 2}$. The sound speed, $\tilde{c}$, is defined less conventionally here to be the square root of the ratio of perturbed pressure to perturbed mass density: $\tilde{c} \equiv\left(p^{\prime} / \rho^{\prime}\right)^{1 / 2}$. We can express the equations of motion in terms of $v$ as follows:

$$
\left(\omega^{2}-k_{z}^{2} v_{\mathrm{A}}^{2}\right) v=\left[(\boldsymbol{k} \cdot v)\left(\tilde{c}^{2}+v_{\mathrm{A}}^{2}\right)-k_{z} v_{z} v_{\mathrm{A}}^{2}\right] \boldsymbol{k}-v_{\mathrm{A}}^{2} k_{z}(\boldsymbol{k} \cdot \boldsymbol{v}) \hat{z} .
$$

For most of the purposes of this paper, the energy equation may be left unspecified, and $\tilde{c}$ may be assumed constant. However, we use the energy equation when we discuss slow mode damping at the cooling scale in $\S$ 5.1.1. There we assume that temperature fluctuations decay in a cooling time, $t_{\text {cool }} ;$ more precisely,

$$
i \omega T s^{\prime}=\frac{T^{\prime}}{t_{\text {cool }}} \quad \text { (energy conservation), }
$$

where $s^{\prime}$ is the perturbed entropy per particle and $T$ is the temperature. Combining this equation with the following monatomic ideal gas relations,

$$
\frac{p^{\prime}}{p}=\frac{\rho^{\prime}}{\rho}+\frac{T^{\prime}}{T}=\frac{2}{3} s^{\prime}+\frac{5}{3} \frac{\rho^{\prime}}{\rho}
$$

yields

$$
\tilde{c}^{2} \equiv \frac{p^{\prime}}{\rho^{\prime}}=c_{T}^{2} \frac{2-i 5 \omega t_{\mathrm{cool}}}{2-i 3 \omega t_{\mathrm{cool}}},
$$

where $c_{T}^{2} \equiv p / \rho$ is the square of the isothermal sound speed. When $\omega t_{\text {cool }} \ll 1, \tilde{c}$ is the isothermal sound speed; when $\omega t_{\text {cool }} \gg 1$, $\tilde{c}$ is the adiabatic sound speed.

\section{A1. ALFVÉN MODE}

The Alfvén mode is incompressible, $\boldsymbol{k} \cdot \boldsymbol{v}=0$. Thus, the term involving the sound speed in the momentum equation vanishes, and the properties of the Alfvén mode are independent of the equation of energy conservation. We obtain the dispersion relation

$$
\omega=v_{\mathrm{A}}\left|k_{z}\right|,
$$

by forming the cross product of equation (A4) with $k$. The eigenfunction satisfies

$$
n^{\prime}=\boldsymbol{k} \cdot \boldsymbol{v}=\hat{\boldsymbol{z}} \cdot \boldsymbol{v}=0, \quad \boldsymbol{b}=-\operatorname{sign}\left(k_{z}\right) \boldsymbol{v} .
$$

Note that both $v$ and $\boldsymbol{b}$ are perpendicular to $\hat{z}$ : the Alfvén wave is polarized transverse to the unperturbed magnetic field.

\section{A2. SLOW MODE}

We summarize the properties of the slow mode to lowest order in $k_{z} / k \ll 1$, the limit appropriate to the MHD cascade. To obtain the dispersion relation, we assume, subject to verification, that the perturbation in total pressure (i.e., thermal plus magnetic pressure) vanishes to second order in $k_{z} / k$. Note that the perturbation in total pressure is proportional to the terms in parentheses in equation (A2) and in square brackets in equation (A4). From the vanishing of this pressure term in equation 
(A4), we then have

$$
\boldsymbol{k} \cdot \boldsymbol{v}=\frac{v_{\mathrm{A}}^{2}}{\tilde{c}^{2}+v_{\mathrm{A}}^{2}}\left[1+O\left(\frac{k_{z}}{k}\right)^{2}\right] k_{z} v_{z} .
$$

The $z$-component of equation (A4), with $\boldsymbol{k} \cdot \boldsymbol{v}$ given by equation (A10), then yields the dispersion relation

$$
\omega=\frac{\tilde{c}}{\sqrt{\tilde{c}^{2}+v_{\mathrm{A}}^{2}}}\left[1+O\left(\frac{k_{z}}{k}\right)^{2}\right] v_{\mathrm{A}}\left|k_{z}\right| .
$$

Next, we solve for the eigenfunction to lowest order in $k_{z} / k$ :

$$
\frac{n^{\prime}}{n} \simeq \operatorname{sign}\left(k_{z}\right) \frac{v_{\mathrm{A}} v_{z}}{\tilde{c} \sqrt{\tilde{c}^{2}+v_{\mathrm{A}}^{2}}}, \quad v_{x} \simeq-\frac{\tilde{c}^{2}}{\tilde{c}^{2}+v_{\mathrm{A}}^{2}} \frac{k_{z}}{k} v_{z}, \quad v_{y}=0, \quad \boldsymbol{b} \simeq-\operatorname{sign}\left(k_{z}\right) \frac{\tilde{c} v}{\sqrt{\tilde{c}^{2}+v_{\mathrm{A}}^{2}}},
$$

where the $x$-axis has been chosen to lie in the plane containing $\boldsymbol{k}$ and $\hat{z}$. We now see that our assumption of negligible perturbed pressure is self-consistent to lowest order in $k_{z} / k$. While we have not used the $x$-component of equation (A4), both the left-hand side and the right-hand side of this equation are of the same order in $k_{z} / k$.

The vanishing of the slow mode's perturbed pressure may be understood as follows: the period of the slow mode is comparable to the time for a disturbance to cross a parallel wavelength at the slower of $\tilde{c}$ and $v_{\mathrm{A}}$. Total pressure balance is set up within the time that the fast mode, which travels at the faster of $\tilde{c}$ and $v_{\mathrm{A}}$, crosses a wavelength. This may be seen by solving equation (A4) exactly (treating $\tilde{c}$ as a constant): the slow mode's perturbed pressure is negligible, and our approximate solution is valid, when $2\left(k_{z} / k\right) \tilde{c} v_{\mathrm{A}} /\left(\tilde{c}^{2}+v_{\mathrm{A}}^{2}\right) \ll 1$, which implies that $k_{z} \min \left(\tilde{c}, v_{\mathrm{A}}\right) \ll k \max \left(\tilde{c}, v_{\mathrm{A}}\right)$.

Note that both $v$ and $b$ are nearly parallel to $\hat{z}$ : the slow wave polarization is nearly aligned with the unperturbed magnetic field. Moreover, in the limit that $\tilde{c} \gg v_{\mathrm{A}}$, the slow mode is nearly incompressible.

\section{A3. ENTROPY MODE}

The linearized MHD equations yield four modes; in addition to the Alfvén, slow, and fast modes, there is a lesser known mode: the entropy mode. For adiabatic fluid motions, the energy equation is $\omega s^{\prime}=0$. The entropy mode has $\omega=0$, and its eigenfunction is given by $p^{\prime}=\boldsymbol{v}=\boldsymbol{b}=0, T^{\prime} / T=-n^{\prime} / n=2 s^{\prime} / 5$.

In the following, we calculate the eigenvalue and eigenvector of the entropy mode in the presence of cooling, i.e., when the energy equation is given by equation (A5). We shall see that the entropy mode decays in a time comparable to $t_{\text {cool }}$.

We consider only wavenumbers sufficiently large that the fast mode's crossing time is much faster than the entropy mode's decay time, i.e., $k \gg k_{\text {fast }} \equiv\left(\tilde{c}^{2}+v_{\mathrm{A}}^{2}\right)^{-1 / 2} t_{\text {cool }}^{-1}$. Therefore, the total pressure perturbation vanishes. Note that $k>k_{\mathrm{fast}}$ in the vicinity of the cooling scale (eq. [13]), where the present calculation is most relevant.

The dispersion relation may be obtained from equation (A4), after setting the perturbed total pressure (the term in square brackets) to zero. The result is equation (A11), which has three roots: two counterpropagating slow modes and the entropy mode. To solve for the entropy mode, it is convenient to rewrite equation (A11) as follows:

$$
i \omega t_{\mathrm{cool}}=\frac{4+2 \beta\left[1-\left(v_{\mathrm{A}} k_{z} / \omega\right)^{2}\right]}{6+5 \beta\left[1-\left(v_{\mathrm{A}} k_{z} / \omega\right)^{2}\right]},
$$

where $\beta \equiv 2 c_{T}^{2} / v_{\mathrm{A}}^{2}$, and where equation (A7) has been used for $\tilde{c}$. Presupposing that $\omega$ is of order $t_{\mathrm{cool}}^{-1}$, we see that the right-hand side of this equation has two limiting behaviors, depending on the value of $k_{z}$.

On small scales, where $\min \left(v_{\mathrm{A}}, c_{T}\right) t_{\text {cool }} k_{z} \gg 1$,

$$
\omega=-i \frac{2}{5} \frac{1}{t_{\text {cool }}}
$$

In this limit the slow mode crossing time is faster than cooling, so the thermal pressure perturbation vanishes, as may be seen directly from equation (A7). Thus, to lowest order in $\left(k_{z} v_{\mathrm{A}} t_{\text {cool }}\right)^{-1}$, the eigenfunction is the same as for the adiabatic case, $p^{\prime}=\boldsymbol{v}=\boldsymbol{b}=0, T^{\prime} / T=-n^{\prime} / n=2 s^{\prime} / 5$.

On large scales, where $k_{z} v_{\mathrm{A}} t_{\text {cool }} \ll 1$, equation (A13) implies that

$$
\omega=-i \frac{4+2 \beta}{6+5 \beta} \frac{1}{t_{\text {cool }}}, \quad v_{\mathrm{A}} t_{\text {cool }} k_{z} \ll 1 .
$$

Substituting this into equation (A7) and using total pressure balance yields the eigenfunction $n^{\prime} / n=b_{z} / v_{\mathrm{A}}=-\left(p^{\prime} / p\right)(\beta / 2)$. The remaining nonzero components of the eigenfunction are $T^{\prime} / T=-\left(n^{\prime} / n\right)(1+2 / \beta)$ and $s^{\prime}=-\left(n^{\prime} / n\right)(5 / 2+3 / \beta)$.

Armstrong, J. W., Rickett, B. J., \& Spangler, S. R. 1995, ApJ, 443, 209

Balsara, D., \& Pouquet, A. 1999, Phys. Plasmas, 6, 89

Banks, P. M. 1966, Planet. Space Sci., 14, 1105

Barnes, A. 1966, Phys. Fluids, 9, 1483

Braginskii, S. I. 1965, Rev. Plasma Phys., 1, 205

Cho, J., \& Vishniac, E. T. 2000a, ApJ, 538, 217 .2000b, ApJ, 539, 273

\section{REFERENCES}

Goldreich, P. 1998, AAS, 192, 4603

Goldreich, P., \& Sridhar, S. 1995, ApJ, 438, 763

-. 1997, ApJ, 485, 680

Higdon, J. C. 1984, ApJ, 285, 109

. 1986, ApJ, 309, 342

Higdon, J. C., \& Conley, A. 1998, AAS, 192, 4609

Lambert, H. C., \& Rickett, B. J. 2000, ApJ, 531, 883 


\section{LITHWICK \& GOLDREICH}

Maron, J., \& Goldreich, P. 2001, ApJ, 554, 1175

Monin, A. S., \& Yaglom, A. M. 1971, Statistical Fluid Mechanics (Cambridge: MIT Press)

Müller, W., \& Biskamp, D. 2000, Phys. Rev. Lett., 84, 475

Quataert, E. 1998, ApJ, 500, 978

Rickett, B. J. 1977, ARA\&A, 15, 479

Rickett, B. J. 1990, ARA\&A, 28, 561

Spitzer, L., Jr. 1978, Physical Processes in the Interstellar Medium (New York: John Wiley \& Sons)

Sridhar, S. 1998, Conf. Interstellar Turbulence, Puebla, Mexico

Tennekes, H., \& Lumley, J. L. 1972, A First Course in Turbulence (Cambridge: MIT Press) 